\title{
Parametrization of Survival Measures, Part I: Consequences of Self-Organizing
}

\author{
Oliver Szasz, Andras Szasz \\ Biotechnics Department, Faculty of Mechanical Engineering, St. Istvan University, Budaors, Hungary \\ Email: biotech@gek.szie.hu
}

How to cite this paper: Szasz, O. and Szasz, A. (2020) Parametrization of Survival Measures, Part I: Consequences of SelfOrganizing. International Journal of Clinical Medicine, 11, 316-347. https://doi.org/10.4236/ijcm.2020.115031

Received: April 9, 2020

Accepted: May 24, 2020

Published: May 27, 2020

Copyright $\odot 2020$ by author(s) and Scientific Research Publishing Inc. This work is licensed under the Creative Commons Attribution International License (CC BY 4.0).

http://creativecommons.org/licenses/by/4.0/

(c) (i) Open Access

\begin{abstract}
Lifetime analyses frequently apply a parametric functional description from measured data of the Kaplan-Meier non-parametric estimate (KM) of the survival probability. The cumulative Weibull distribution function (WF) is the primary choice to parametrize the KM. but some others (e.g. Gompertz, logistic functions) are also widely applied. We show that the cumulative twoparametric Weibull function meets all requirements. The Weibull function is the consequence of the general self-organizing behavior of the survival, and consequently shows self-similar death-rate as a function of the time. The ontogenic universality as well as the universality of tumor-growth fits to WF. WF parametrization needs two independent parameters, which could be obtained from the median and mean values of KM estimate, which makes an easy parametric approximation of the KM plot. The entropy of the distribution and the other entropy descriptions are supporting the parametrization validity well. The goal is to find the most appropriate mining of the inherent information in KM-plots. The two-parameter WF fits to the non-parametric KM survival curve in a real study of 1180 cancer patients offering satisfactory description of the clinical results. Two of the 3 characteristic parameters of the KM plot (namely the points of median, mean or inflection) are enough to reconstruct the parametric fit, which gives support of the comparison of survival curves of different patient's groups.
\end{abstract}

\section{Keywords}

Self-Organizing, Self-Similarity, Avrami-Function, Weibull-Distribution, Survival-Time, Allometry, Entropy, Bioscaling

\section{Introduction}

The driving force of the overall spontaneous progressions in nature is the at- 
tempt to minimize the actual energy and maximize the entropy in the actual processes. In this sense, life follows the basic thermodynamic laws: the living process continuously "burns" the incoming "nutrition". Only the energy-pump of the incoming sun-energy makes the difference: creates original gradients which are later divided into other inhomogeneities by spontaneous processes.

Life process tries to diminish the working energy of the sunlight by increasing the overall entropy of the environment. Living process lowers the electron energy by the oxidation producing outgoing (waste) final "products". The gradual loss of electron energy of the "nutrition" molecules is the energy to sustain life. Simply speaking, the living process is a dissipative entropy producer. As the Nobel laureate physiologist A. Szentgyorgyi states "Life is nothing but an electron looking for a place to rest" [1].

Living objects are open systems among various environmental surroundings, adapting themselves to the conditions around, forming self-organized structures [2], and forcing evolution [3]. The approach of complexity becomes a useful tool for the description of nature [4] [5]. Self-organization appears in various scientific problems [6]. The self-organization explains multiple structural and dynamical challenges in biology [7]; it is observed in broad range of research from the gene-regulatory networks [8], through the cells [9], to the general evolution of living objects [10].

The invariance of magnification (scale invariance, when the up or down magnification shows similar structures) is the form of self-similarity, which is a typical consequence of the self-organizing processes, [11] [12]. It has developed a new discipline, the fractal physiology [13] [14] [15]; where stochastic processes are applied instead of the deterministic actions, so the predictions of the distant future always have random, unpredictable elements.

Random stationary, stochastic, self-organizing processes form dynamic behaviors [16], define a spatiotemporal-fractal structure, which is self-similar both in space and time [17]. The spatiotemporal fractal structure is a fingerprint of the self-organizing [18], and especially characteristic for the living matter [19]. The basal metabolism as the energy consumption of the living objects has a central role in system biology [20] and describes the biosystems by definite properties [21]. According to the system biology:

- life is complexly organized in a wide range of magnification and different levels of interactions,

- life is self-regulated with various feedback processes,

- the living systems are open, dissipative objects with multilevel interactions with the environment,

- the activity of life processes has intensive cross-talks of different levels of its organization,

- the specific forms and properties are complexly environment dependent These points are important for the universality of life, for the dynamic fluctuations and scaling too [22], and as a character of life, it could be used in its di- 
agnosis as well [23]. Dynamical interactions have a spatiotemporal fluctuation which also has a scaling behavior. Homeostatic time-fluctuation is the so-called pink noise [24], that characterizes the noise of homeostasis.

The above complex biological processes connect to the biological allometry, scaling, non-equilibrium, and non-linear thermodynamics. Special self-similarity characterizes the mass-allometry by universal scaling, and it appears in a large category of living structures and processes [25], which rigorously optimizes the metabolic power in a universal frame, [26]. Scaling is a simple power function, (like $\left.P(x)=a x^{b}\right)$, where $a$ and $b$ are constants, therefore the form of $P(x)$ remains the same during any magnification of $x$. This scaling condition characterizes the biomaterials, which is indeed scaled universally on a very wide range of magnifications from the subcellular energy-consumption through mitochondria and respiratory complexes to the largest animals by scaling exponent $\alpha=$ $3 / 4$, [27]. The fingerprint of complexity can be found in various fields of biology, showing unified principles of self-organization [28]. Note, that mitochondria probably has a key-role in this complex behavior of living objects, because the non-mitochondrial respiration scaling factor is lower, $(\alpha=2 / 3)$, characterizing the simple surface-volume ratio in these processes, [29], however the robust category of living systems is scaled by complex manner [30]. Different conditions modify the power function [31], forming various universality classes by self-similarity.

Self-organized processes are widely investigated in solid-state reactions (precipitations, phase-transitions, aggregations, nucleation, growth, etc.). The theory of phase-transition involving simultaneous random nucleation and growth was pioneered by Kolmogorov [32], Johnson, Mehl [33] and Avrami [34]. It is called Johnson-Mehl-Avrami-Kolmogorov (JMAK) model, revised later by others, [35], [36]. It describes the kinetics of phase transformation when nucleation is spatially random. The JMAK theory and one of its formulation called Avrami-function $(\mathrm{AF})$ were introduced for solids to serve as mathematical models of different biological processes, [37] [38] and even for DNA replication process, [39] too. Experimental data [40] [41] [42] [43], prove a certain universality of the Avrami-equation to describe the real processes, which could be a useful tool for further research, [44]. It is generally useful for studying different processes with no known special system parameters, similarly to the critical phenomena of the physical-laws near to the phase-transition [45].

The $\operatorname{AF}(A(t))[46]$ in its most applicable forms:

$$
\begin{aligned}
& \ln (-\ln (A(t)))=n \ln (t)+\ln \kappa \\
& A(t)=1-\exp \left(\kappa t^{n}\right)
\end{aligned}
$$

where $t$ is the elapsed time of the process, $\kappa$ depends linearly on the nucleation rate and on the growth-rate by the power of three. The so called "Avrami constant" ( $n$ ) was introduced in simple model $n=4$, and so originally in solids it was considered an integer [47]. It is interesting, that the space-fractal dimension 
dependens on AF [48]. Here $n$ value is not necessarily an integer and depends well on the processes that are described by it. The fractal dimension, and the power-law of self-similarity are tightly connected [49]. Experimental data show, that the progression of many reactions in biology also follow the $A(t) \mathrm{AF}$ with various, non-integer characteristic constants [40]. It was observed universally in different processes from a wide range of structural and dynamical situations of living systems [44] [50] [51] [52].

The non-equilibrium thermodynamical formalism could be applied to a selforganized system of malignancy in space and time [53]. Cancer breaks the network of normal cells, while the cooperative tissue harmony changed to non-cooperative competitiveness forms a new complex structure non-linearly far from the thermodynamic equilibrium. Cancer could be described as a dynamical phase transition from healthy to cancerous [54], described with a clear analogy with phase transitions in a lifeless nature. Starting with an avascular situation and forming a dormant microscopic cluster [55], it continues to develop new angiogenetic formations by epithelial-mesenchymal cell transition, induced by bioelectromagnetic forces, [56]. Tumor leaves the dormant state by an allometric transformation [57], and the previously almost undetectable phase becomes traceable. An Avrami-like function in time describes its development [58]. This idea was used to show the validity of Avrami description [59] and extended to metastases while studying the transition of avascular appearance of tumorous clusters [60] to vascular phase, which bases the dissemination of malignant cells, [61]. Metastases are developed by a first order phase transition of cells from non-cancerous to metastatic ones [62]. The development of this new phase needs a great amount of energy. The energy dissipates in the system, produces a high rate of entropy development.

The general transport structure (blood-vessel network) of the tissues forms fractals by allometric scaling, including the angiogenetic processes in tumor formation [57]. In oncological applications, the available metabolic transport and the fractal dimensions of the angiogenetic network determine the average survival of a tumor. The average survival of the tumor-cells shortens by the growing fractal dimension of the transport network and modified by some kind of an alimentation of the tumor, [63]. The tumor-growth follows the universal law of scaling [64], which can be used in cancer-research [65].

The dynamics of the evolution of cancer produces various phases of the growing structures due to the genetic instability, leading to phase transitions [66]. Tumor development operates near the threshold of phase transition, destabilizing the actual structure, making it highly heterogeneous [67], producing a large variety of random mutations [68], finding the most optimal conditions of the further proliferation. Their development is based on competition, a "fight" for the individual survival. The optimal strategy is well known in the game-theory [69] where the mixed-strategy forms Nash equilibrium in the non-cooperative game by random variation behind [70]. This situation is typical for topological phase transi- 
tions [71], where the cooperation emerges despite the selfish, non-cooperative individual participating cells [72].

Our objective in this article is to find a parametric description of overall survival, which fits the self-organized processes and able to show the inherent information of survival measurements of cancer patients.

\section{Method}

Most of the survival analyses in medical evaluations use the Kaplan-Meier (KM) non-parametric estimator [73] [74], used for incomplete observations. KM is useful to examine the probability of lifetime and effectivity of the chosen treatment for such lethal diseases like cancer. The computed probability of an event in a definite point of time:

$$
\begin{aligned}
& \left(\begin{array}{c}
\text { Probability at actual } \\
\text { time of observation }
\end{array}\right) \\
& =\frac{\left(\begin{array}{c}
\text { Number of participants living } \\
\text { at the strat of observation }
\end{array}\right)-\left(\begin{array}{c}
\text { Number of participants died or } \\
\text { censored during the of observation }
\end{array}\right)}{\left(\begin{array}{c}
\text { Number of participants living } \\
\text { at the strat of observation }
\end{array}\right)}
\end{aligned}
$$

$\mathrm{KM}$ estimator is defined by multiplying the above described successive probabilities by any earlier point of time obtaining the final estimate:

$$
K M(t)=\prod_{t_{i} \leq t}\left(1-\frac{d_{i}}{n_{i}}\right)
$$

where $d_{i}$ is the number of deaths at the time $t_{i} ; t_{i}$ is a time when at least one death had happened in the examined cohort, and $n_{i}$ is the number of individuals known to survive (not censored, exists in the study) at time $t_{i}$. Some modifications were done in tails (pessimistic approach when short-tailed) [75], and optimistic approach, a fat-tailed [76] is in use having a difference in survivals at the end of the trial.

The best method for mining data could be when the non-parametric KM survival plot can be parameterized. The description of survival curves by parametric distribution function is a long-term effort [77] allowing the optimization of the information from the measured dataset. For the correct parametrization, we have to take an overview on the scientific facts that we can use for the research of the optimal parametrization. The most important result available is the parametric solution that is connected to the spatiotemporal self-organization and the self-similarity of developed structures.

The parametrization of survival measures we use to the universality of life consideres its self-organized self-similarity. The progression of life involves nonlinear and non-equilibrium thermodynamical consequences including the fractal description and similar processes of the phase transitions in non-living systems. For calculating the survival-time, let $T$ be the stochastic variable defined on the set of individuals, (lifetime). The lifetime distribution function is the probability 
of the lifetime being less than or equal to $t$, namely

$$
p_{L}(t)=P\{T \leq t\}
$$

Thus, the survival probability distribution (survival function) can be defined by the probability of the $T$ lifetime being higher than $t$, that can be expressed in the form of

$$
p_{S}(t)=1-p_{L}(t)=P\{T>t\}
$$

The density function of the lifetime distribution function is the

$$
f(t)=\frac{\mathrm{d} p_{L}(t)}{\mathrm{d} t}
$$

probable density, therefore, the average lifetime is:

$$
\langle T\rangle=\int_{0}^{\infty} t f(t) \mathrm{d} t=\int_{0}^{\infty} p_{S}(t) \mathrm{d} t
$$

Introducing the $h(t) \mathrm{d} t$ death rate is the probability that in case of a $t$ length survival time, death occurs at $(t+\Delta t)$ and $(h(t)$ is the "hazard function" or "death rate"). Therefore, the probability is that in the case of a $t$ length time survival, death occurs at $(t+\Delta t)$ is

$$
h(t) \Delta t=1-\frac{p_{S}(t+\Delta t)}{p_{S}(t)}=-\frac{\mathrm{d} \frac{p_{S}(t)}{\mathrm{d} t}}{p_{S}(t)} \Delta t=-\frac{\frac{\mathrm{d}\left[1-p_{L}(t)\right]}{\mathrm{d} t}}{p_{S}(t)} \Delta t=\frac{f(t)}{p_{S}(t)} \Delta t
$$

From this:

$$
h(t)=-\frac{\mathrm{d} \frac{p_{S}(t)}{\mathrm{d} t}}{p_{S}(t)}=\frac{f(t)}{p_{S}(t)}
$$

It's cumulative form is

$$
H(t)=\int_{0}^{t} h(\tau) \mathrm{d} \tau=-\ln \left(p_{S}(t)\right)
$$

or

$$
p_{S}(t)=\mathrm{e}^{-H(t)}
$$

Biological systems are strictly self-organized [78]. The inherent property of the living objects is the self-organizing and the consequent self-similarity of the living structures [11], which could be the basis of the proper parameterization of survival.

Taking the self-similarity into consideration, death-rate (failure rate in (8)) must be a self-similar time function [44], mirrored by a scaling like:

$$
h(t)=\alpha t^{\beta}
$$

Its self-similarity is obvious because it gives the same function by magnification $m$ :

$$
h(m t)=\alpha(m t)^{\beta}=m^{\beta} \alpha t^{\beta}=m^{\beta} h(t)
$$


The survival probability distribution function from (9) and (10) is:

$$
p_{S}(t)=\mathrm{e}^{-\int_{0}^{t} h(\tau) \mathrm{d} \tau}
$$

The self-similar death rate (hazard function) is:

$$
H(t)=\int_{0}^{t} \alpha \tau^{\beta} \mathrm{d} \tau=\frac{\alpha}{\beta+1} t^{\beta+1}
$$

Substituting (14) with survival (13), we get:

$$
p_{S}(t)=\mathrm{e}^{-\int_{0}^{t} \alpha \tau^{\beta} \mathrm{d} \tau}=\mathrm{e}^{-\frac{\alpha}{\beta+1} t^{\beta+1}}
$$

Introducing

$$
t_{0}=\left(\frac{n}{\alpha}\right)^{1 / n} \text { and } n=\beta+1
$$

Hence:

$$
p_{S}(t)=\mathrm{e}^{-\left(\frac{t}{t_{0}}\right)^{n}}
$$

which has two parameters for one curve, $t_{0}$, is the scale parameter, which is the natural scale of the time-function variation, and $n$ is the shape parameter. Consequently, the lifetime distribution function $p_{L}(t)$, by (3) and (4) is the wellknown $\mathrm{AF}(A(t))$ or cumulative form of the two-parametric cumulative Weibull distribution $(W(t))$ :

$$
p_{L}(t)=A(t)=W(t)=1-\mathrm{e}^{-\left(\frac{t}{t_{0}}\right)^{n}}=1-p_{S}(t)
$$

with additional conditions $t \geq 0, A(t)=W(t)=0$, when $t<0$. The inverse function, when the $t$-time is calculated from a given $\mathrm{p}$ probability is:

$$
t=W_{\text {inv }}(p)=t_{0}(-\ln (1-p))^{1 / n}
$$

There are various parameters characterizing the WF from the time of development independently. The shape parameter of WF is usually $n>1$, following a sigmoid curve, which form is a psychometric function [79] anyway. In cases when $n \leq 1$ the survival is a simple exponential function with rapid decrease by the decreasing of $n$.

The cumulative Weibull distribution (Weibull function, WF) is highly universal and represents all the features described in the introduction above. The formal identity of WF with the AF in JMAK inherently involves the phase transition approach, and the mechanics follow the tumor kinetics, [59].

The AF and WF have been used for a long time for survival/reliability description. Originally Weibull's statistics was developed to describe the fracture of brittle materials [80], [81] and to calculate the probability of the damage-free survival of the given material. It can be derived from geometric scale invariance (fractal organized structures) by physical principles, [82] in mechanical mills. It is frequently applied in the study of mechanical fatigue and failure [83]. 
The fit of WF to the non-parametric KM is completely rigorous when a strictly homogeneous cohort of patients is investigated, with unified equivalence of the participating individuals followed until the decease or censoring. This grouping selection apparently limits the applicability of WF. The parametrization of the aging and natural death has no such grouping selection, it is related to every human being and their survival. The epidemiological studies in gerontology refer to the Gompertz-distribution, [84]. The Gompertz function (GF) is a function of time. When $G(t)$ represents the number of individuals in the given period of time, $t, G_{0}$ is the number of subjects at the start of the counting time, then GF is:

$$
G(t)=G_{0} \exp (-a \cdot(\exp (b \cdot t)-1))
$$

The parameters $a$ and $b$ are positive and $a$ is connected to the growth, while $b$ is connected to the displacement in variable $t$. GF is also a double-parametric function, similarly to the $n$ and $t_{0}$ in WF.

During the historical development of WF, it has started to characterize the aging of the non-living components and machineries (reliability) while the GF was initially developed for the ageing of living objects [85]. By developing the statistical methods, soon, both the Weibull and Gompertz distribution have started to be applied for description of tumor-development and cancer-death. The comparison of the two distributions shows that the best fit of GF is $(a=9.03, b=2.58)$ and the best fit of WF is $\left(n=1.11, t_{0}=0.04\right)$; $\left(1-r^{2}=3.6 \times 10^{-6}, \mathrm{SE}=0.002\right)$; where $\mathrm{SE}$ is the standard error of the regression estimate minimizing the sum of squares of measured and estimated datapairs. Due to their applicability, the Gompertz and Weibull distributions are both commonly used in biological and engineering reliability investigations [86], [87].

The study of Gompertzian distribution for tumors supports a hypothesis that the fractal structure weakens and, in the end, it disappears by the growth of the tumor [88]. In general, the tumor-growth follows a universality, [64] [89], which prefers to use the WF. The clear fitting of allometric scaling by the fractal structure of the tumor [64] shows not only the tumor growth but the validity of the allometry in the growth of the axillary lymph node involvement in breast cancer [90]. In consequence, we choose to use the WF for modelling the KM plot of the overall survival.

The Gompertz distribution could be obtained by the reduction of the generalized exponential Weibull distribution [91], which formulated in a more general form, proposing to derive both distribution from one single [92] and it is applied for survival data with pretty good results.

The GF does not satisfies the self-similarity (formulated in (11)), and therefore, it is not in harmony with self-organizing biological dynamics, which is a certain character of the harmonized biological development, [2]. This might be the reason, why the WF describes the intrinsic causes of age-related mortality 
better (following the homeostasis in the healthy aging process) while the Gompertz distribution reflects the extrinsic factors [93]. Due to the self-similarity of WF, we expect, that the self-organized biological development of tumors intrinsically developing in a healthy environment from where it derives, prefers the WF to describe the KM in malignant diseases accurately. It is a further support for the primary importance of Weibull distribution, that it is derived from the ontological law, and so it is directly connected to the self-organized structure of the living matter [28]. The self-similarity, as the basic fingerprint of self-organizing is not valid in Gompertz distribution. The "mystery" of Gompertz function is probably the equilibrium between the predictable and unpredictable (chaotic) dynamisms, [94]. Contrary to the exponential origin of GF, the self-similarity (power function) of WF's origin hypothesizes some parallels with the opposite pictures of fractal-like organizations and general scale-free (small-words, [95]) large networks (exponential function). Despite the structural preference of WF, GF also fits well to allometry, represented by power-function [96], shown in the development of rats [97]. Although WF fits very well to the growth function of the general ontogenic model, using the data for rat [98] $\left(r^{2}=0.99965\right.$, $\mathrm{SE}=0.949)$; the fit of GF shows the same result $\left(r^{2}=0.99967, \mathrm{SE}=0.884\right)$ for the same allometric curve. The difference is negligible in this regime of development. In the case of animals with larger masses, the difference is also not significant. It is subtle, favoring only the WF for the description of the best regression fit to the allometric scaling result, using the available data from [98]. (The best WF and GF fits to allometry for cow are $\left(r^{2}=0.99978, \mathrm{SE}=1.021\right)$ and $\left(r^{2}=0.99972, r^{2}=0.99972\right)$, respectively. $)$

WF is successfully applied to the living processes as the psychological function [99], describing the sensing processes well in connection with Weber-Fechner law [100], establishing psychometry [101]. Lifetime estimations are frequently approached by WF [86] and WF is also successfully used for clustering gene expression [102].

WF describes the non-parametric KM plot with appropriate accuracy in gerontology [103] [104]. A mathematical link of natural death-rate, aging and complexity is a fundamental tool of lifetime estimation [105] [106], using time-dependent shape-factor $\left(n \cong 7 /\left|\ln \left(t / t_{0}\right)\right|\right)$ to describe the natural death at the end of life. Cancer-death was also described by WF with time-dependent shape-factor, using a similarity between the fracture survival of brittle materials and the specific survival characteristics of a cohort of cancer patients [107] [108]. In this model the shape factor linearly depends on the time and gives surprisingly accurate fit to the data from the cancer-registers.

Due to its self-similar behavior, fractals could be used for modeling cancer [109], and the KM survival plot divided significantly by fractal dimension shows the prognostic value of the fractal analysis well [110]. Consequently, it is possible to evaluate the various images in oncology by the fractal structure and these images can be characterized by Weibull distribution as well [111]. 
Due to the self-similarity, the parametric distribution generally fits well with the KM plot, and so it is successfully used in oncology [112] [113]. The application of the parametric WF approximating the survival curve is a standard approach for the evaluation of clinical trial data, and so it is established theoretically and practically, [34] [86] [99] [114]. Comparing various parametric fits to KM survival plot, the WF was the most accurate [115]. The model was used to analyze the prognostic factors of the survival of cancer patients, and it was proved in a large retrospective analysis with $n=746$ gastric cancer cases, [116].

Summarizing the above, the self-organizing and the self-similarity are universal laws fingerprinted in the fractal description and can be described by cumulative Weibull distribution. This universality of WF is applied to parametrize the KM plot. Due to the universality, the WF parametric regression fits the KM plot with sufficient accuracy and so determines the KM curve by two parameters $\left(t_{0}\right.$ and $n$ ). On the regression, a considerable improvement could be made by smoothing the KM with the hazard data (patients at risk), [117]. Other improvements of the bivariant fit are also available [118], but for simplicity we use the original WF fit to KM insisting on showing the roots that are the universality of WF in survival investigations. Further smoothing and corrections are additional to the clearly established basis, due to the deviations in real cases.

\section{Results}

The characterization of WF has four special points, the value at $t_{0}$, the mean, the median and the inflection point. The median, the mean and the mode (the maximum point in the distribution function is an inflection point in the cumulative curve) are calculable from the parametric formulas, (see Figure 1):

$$
\begin{aligned}
& \operatorname{median}\left[p_{S}(t)\right]=t_{0}[\ln (2)]^{\frac{1}{n}} \\
& \operatorname{mean}\left[p_{S}(t)\right]=t_{0} \int_{0}^{\infty} \mathrm{e}^{-x} x^{\frac{1}{n}} \mathrm{~d} x=t_{0} \Gamma\left(1+\frac{1}{n}\right) \\
& \operatorname{mode}\left[p_{S}(t)\right]=t_{0}\left[\frac{n-1}{n}\right]^{\frac{1}{n}}
\end{aligned}
$$

The corresponding probabilities when $t_{0}=1$ and $n=2$, are $0.5,0.607$ and 0.456 for the median, mode and mean, respectively. The quantile of this function is $\approx 0.632$ and it independent from $n$ value. Limit $\lim _{n \rightarrow 0} p_{S}(t)=0$ through a step-function at $t=0$, while $\lim _{n \rightarrow \infty} p_{S}(t)$ is a step function at $t=t_{0}$, (Figure 2 ). All the noteworthy points are proportional to $t_{0}$, so the natural units of the elapsed time are $t_{0}=1$, when the single $n$-parameter defines the function. The hazard function (9) is constant when $n=1$ (or $\beta=0$, which means the parameter has no effect on the hazard), and it is increasing and decreasing when $n>1$ (meaning the event is more likely to occur) and $n<1$, (meaning the event is less likely to occur), respectively. The $\operatorname{limit} \lim _{n \rightarrow 0} H(t)=0$ is a step-function at $t$ $=0$, and $\lim _{n \rightarrow \infty} H(t)$ is a step function at $t=t_{0}$, (Figure 2). 


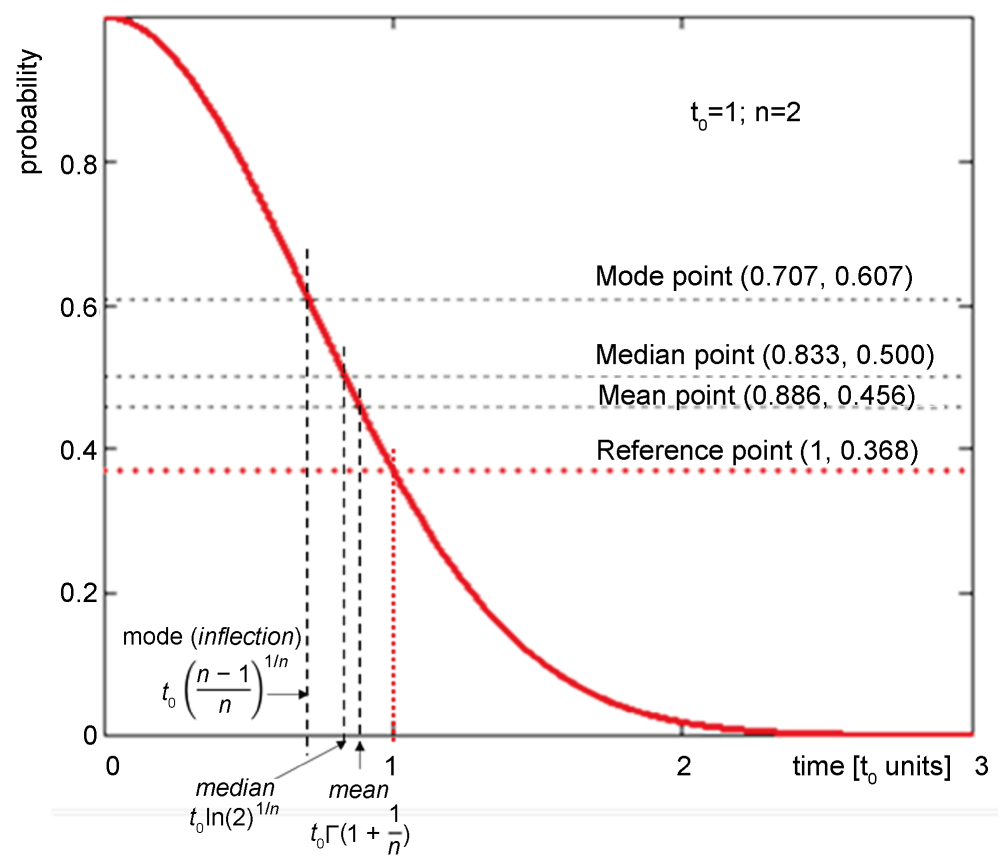

Figure 1. The noteworthy points of the Avrami-Weibull function, when $t_{0}=1$ and $n=$ 2. The reference point of the Avrami-Weibull function is the value $(1 / \mathrm{e} \approx 0.37)$, where $t=t_{0}$. The inflection point marks the mode of the distribution, which is the most frequent probability. When $t_{0}=1$ is chosen, it will be the unit of the elapsed time.

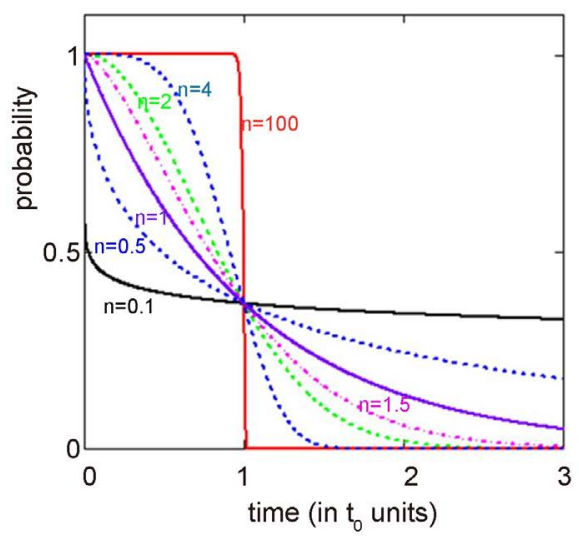

(a)

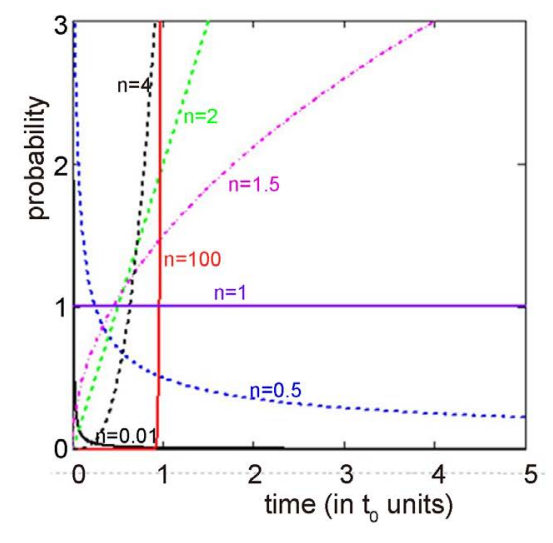

(b)

Figure 2. The limits of (a) survival $\left(p_{S}(t)\right)$ and (b) hazard $(H(t))$ functions $\left(t_{0}=1\right)$.

The various parameter-pairs of WF are shown in Figure 3.

The inflection point in the WF (cumulative Weibull distribution) is the mode of the probability distribution function. It is the most likely appearing value in the Weibull probability distribution function. The inflection in the WF of survival divides the speed of developing death, which reaches its maximum at this point and the transfer of inflection is slowed by the elapsed time.

Programming calculates the result or makes it graphical (Figure 4). This makes it possible to generate the Weibull fit for the Kaplan-Meier routinely by knowing its median and mean values. 


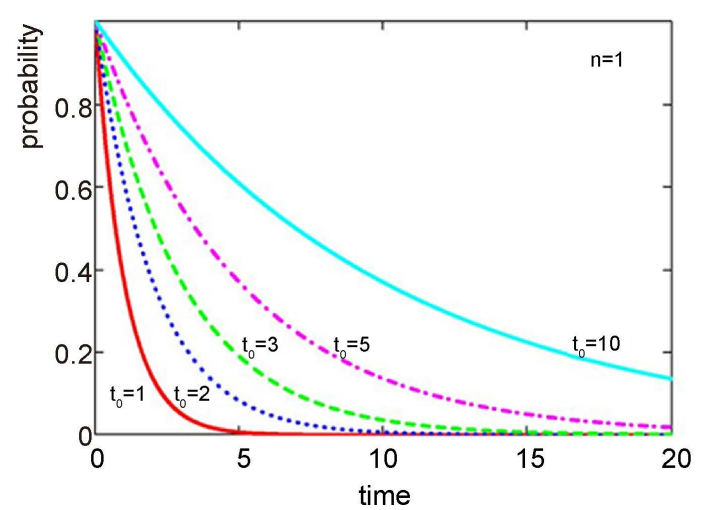

(a)

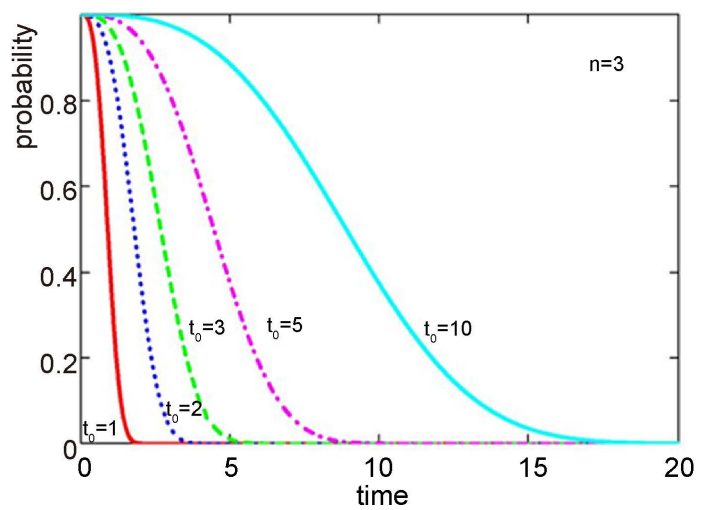

(b)

Figure 3. WF with various parameters (a) changing $t_{0}$ (scale parameter) at constant $n=1$ (shape parameter); (b) $n=3$.

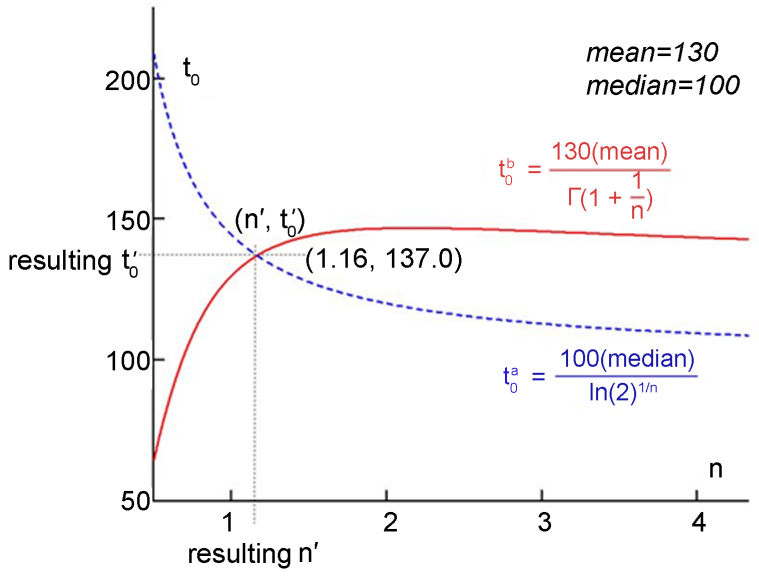

(a)

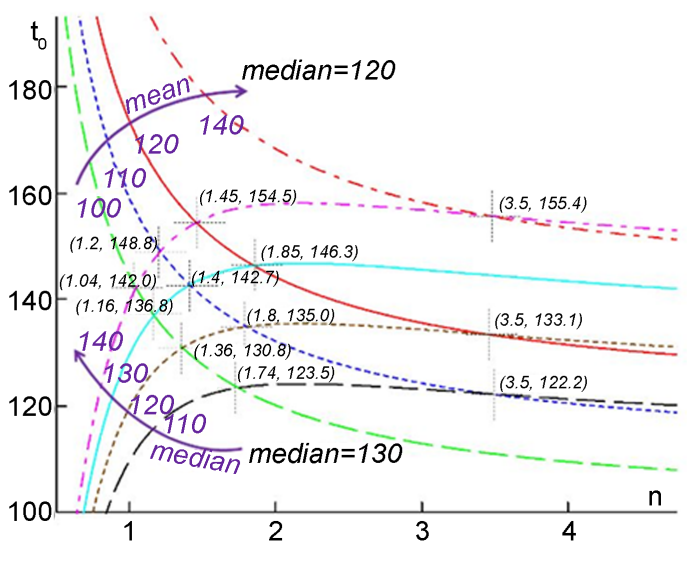

(b)

Figure 4. Graphical solution of reestablishing the Weibull parametric survival curve from the median and mean values. (a) example: $t_{0}^{a}$ and $t_{0}^{b}$ are the time curves from median and mean expressions, respectively. Their common point (crossing) gives the $t_{0}$ and $\mathrm{n}$ parameters of the WF, which-in this case is when the mean is 130 and median is 100, the $t_{0}^{\prime}=137$ and $n^{\prime}=1.16$; therefore, it looks like this: $W(t)=\mathrm{e}^{-\left(\frac{t}{t_{0}}\right)^{n}}=\mathrm{e}^{-\left(\frac{t}{137}\right)^{1.16}}$. (b) a few solutions to show the trend of the graphical results.

The data at the particular points vs. $n$ are shown in (Figure 5 ). The mode changes rapidly in the interval of $n(1,2)$, so reading accurately is difficult, therefore the median and mean are proposed to reestablish the entire WF. However, in a value of $n \approx 3.35$ at $t_{0}=1$ the values of mode, mean and median are practically identical, so the WF could be characterized with a single parameter. Increasing $t_{0}$ does not lead to a significant change of the situation, so in virtually every case, we may approach WF only with one parameter over $n \approx 3.35$.

In conclusion from the above, the parametric regression KM is universally determined by two parameters (the shape parameter $(n)$ and the scale parameter $\left(t_{0}\right)$ of WF), due to the basic behaviors of living processes: their self-organizing 


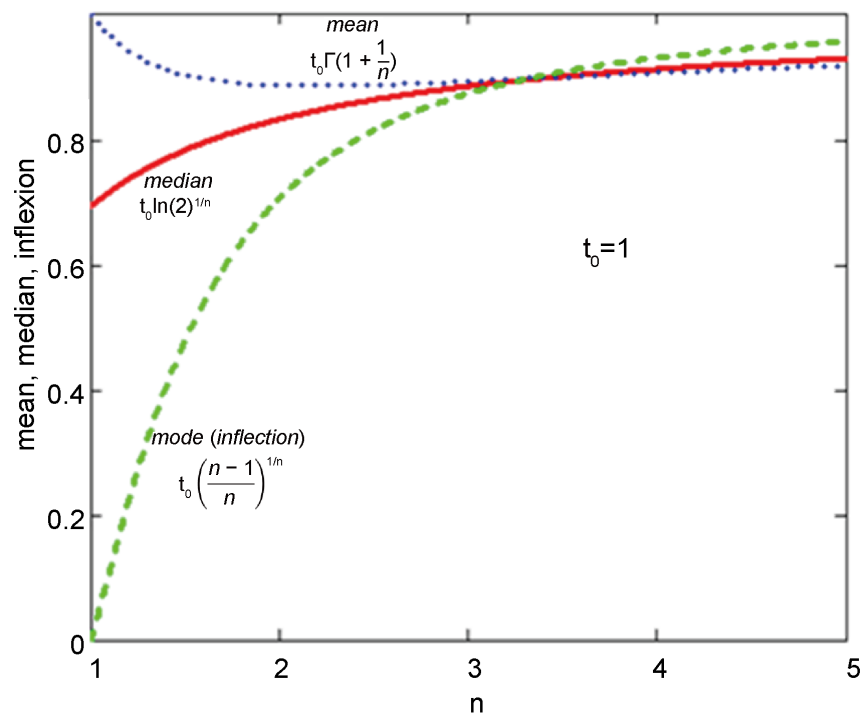

(a)

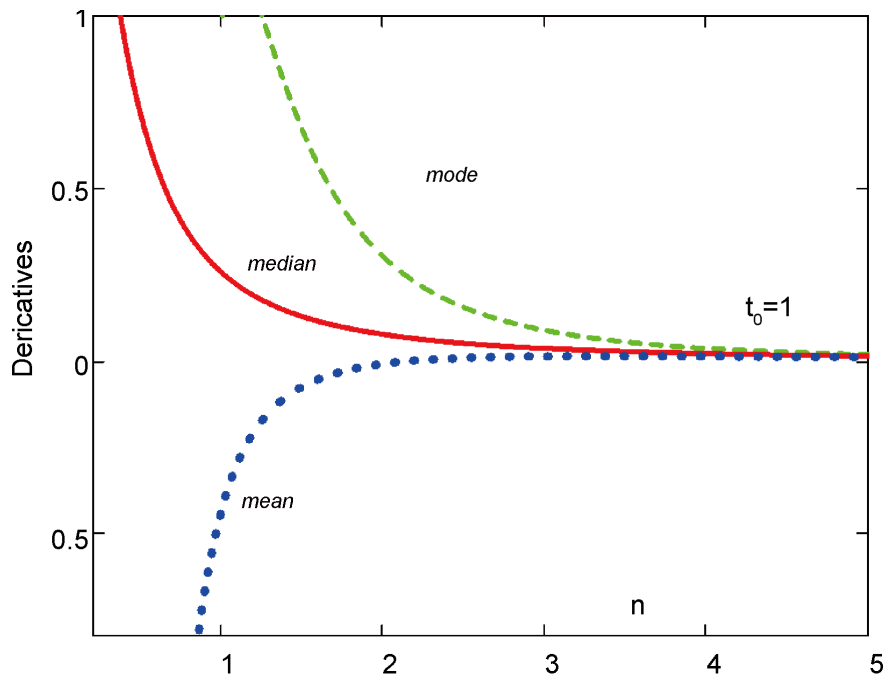

(b)

Figure 5. Characteristics by shape-parameters at $t_{0}=1$. (a) function vs. $n$; (b) derivatives vs. $n$.

and self-similarity, which is characterized well by their spatio-temporal fractal structure. When a clinician tries to describe the main info of the KM survival curves, takes the median value of survival, as a significant parameter characterizing the actual survival result into account. This is, in fact, an automatic characterization by a single parameter of the non-parametric estimation. However, the median alone cannot characterize the long tail of the KM plot; it does not consider the history of the patients in the remaining second half of the cohort, which could be essential for measuring the "cured" [119] anyway. Studying the median alone disregards the real measurable success at the end of the study. Correcting this "mistake" the average (mean) of the KM non-parametric distribution is considered. The mean is affected more by the "tail" of the distribution, so it gives a more accurate idea on the cure rate. The median is more responsible 
for the information about the rapidity of the loss of the patients, while the mean has more part in the information about the length of the effect of the high-success patients, Figure 6.

Sometimes the inflection of $\mathrm{KM}$ is studied too, having the highest death-rate in the study at that point. All are important for characterization, but two of them are independent, and the third could be calculated from the chosen two. The distribution curve must be characterized by two parameters at least.

Two of the three noteworthy points (median, mean, inflection) of the KM may parametrize the non-parametric plot. Measuring or guessing these characteristic points (mainly the median and mean) is a standard comparison of the KM-plots and usually accepted as the result of the actual study. These points really characterize the non-parametric distribution and give the possibility to parametrize, so, in fact, this is a "hidden" parameterization of the KM plot by WF.

A simple approach of Weibull fit could be made on the KM plot by its derivative in the $t_{0}$ reference point, which is proportional to $-n$. (The derivative there is exactly $\frac{\mathrm{d} W\left(t_{0}\right)}{\mathrm{d} t}=-\left(\frac{1}{\mathrm{e}}\right) \frac{n}{t_{0}} \cong-0.368 \frac{n}{t_{0}}$.) Therefore, the parametric evaluation could be checked well at the $t=t_{0}$ point, and the complete parametrization could be established approximately by the value of the $t_{0}$ point and the value of its slope, Figure 7.

The regression could be simplified to linear by double logarithmic approach:

$$
\ln [-\ln (W(t))]=n \ln \left(\frac{t}{t_{0}}\right)=n \ln (t)-n \ln \left(t_{0}\right)
$$

The regression is shown in Figure 8. Note, that this approach is less precise than the function fit, because the double logarithm suppresses the accuracy in real KM fit.

However, the obvious deviation of the regressions from the measured OS is in

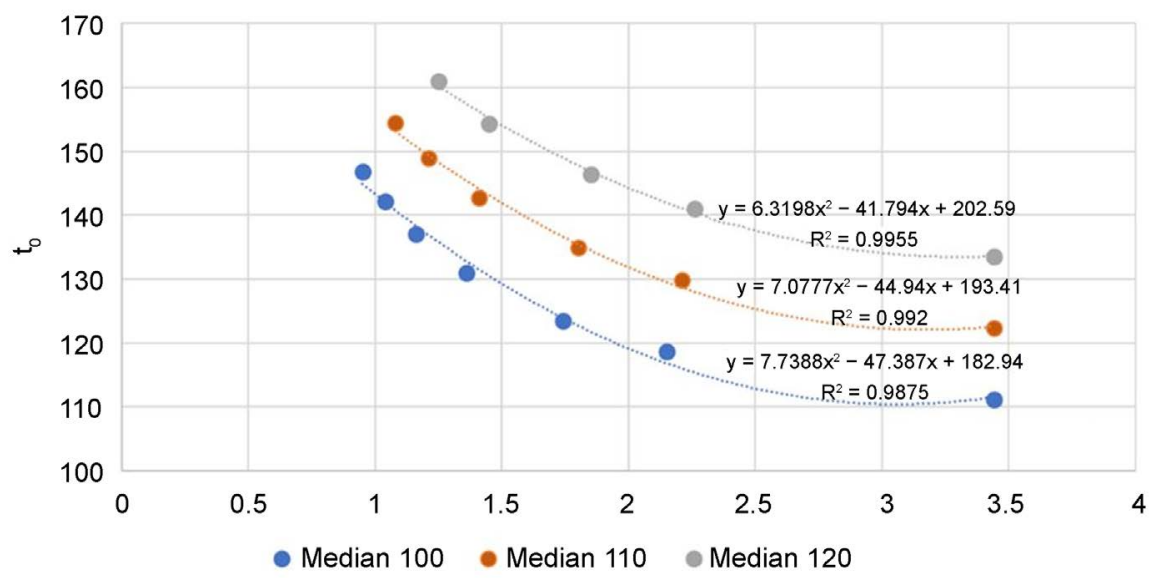

Figure 6. The mean and median changes according to the $n$ and $t_{0}$ parameters. Parabola fits rather well, which connects the two parameters ( $n$ and $t_{0}$ ) at different medians and means. 


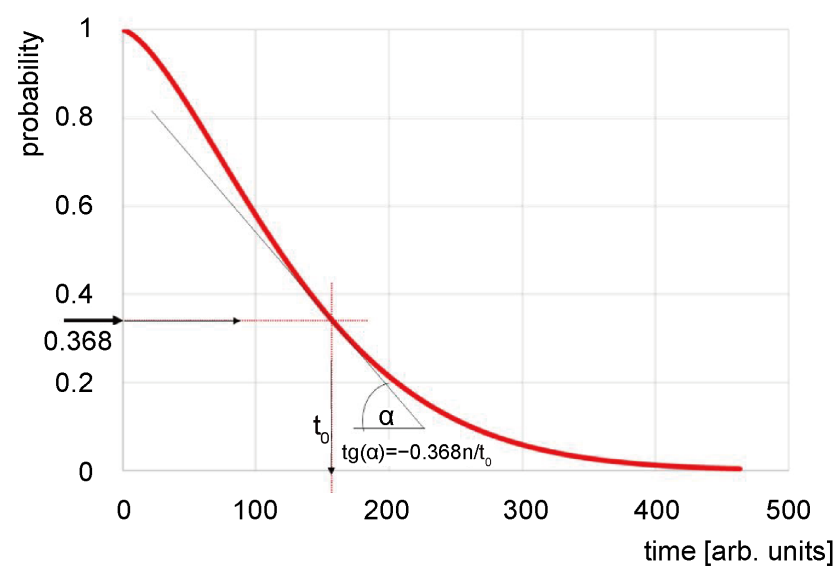

Figure 7. A quick check of the parameters of the Weibull-fit to KM. real process on a $\mathrm{KM}\left(n=1.5, t_{0}=150\right)$.
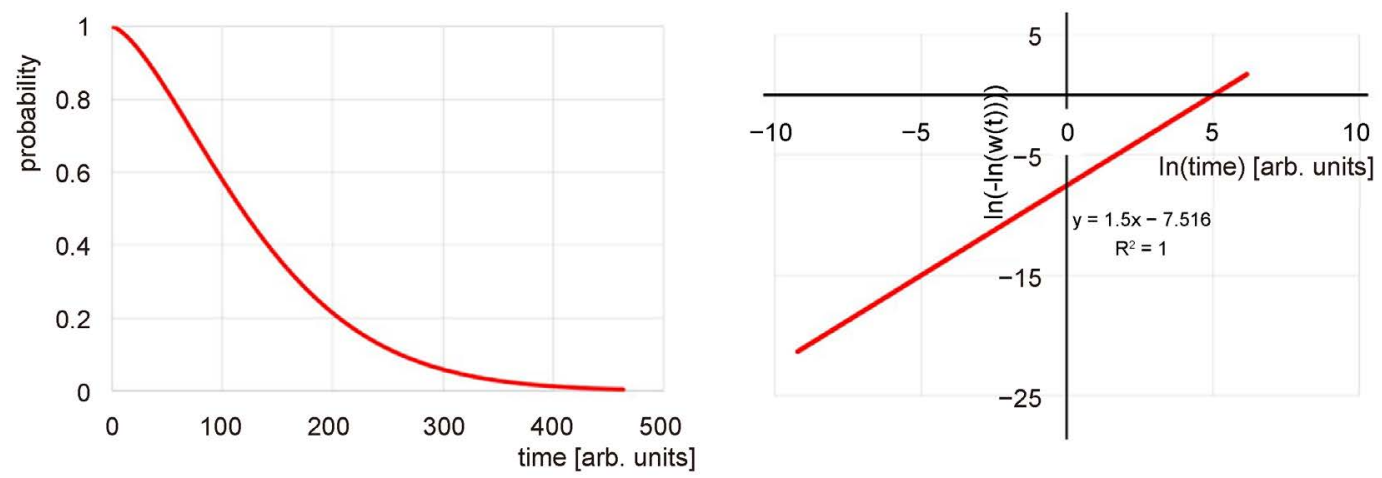

Figure 8. Logarithmic determination of the Weibull parameters $\left(n=1.5, t_{0}=150\right)$. (a) original WF, (b) $\ln [-\ln (W(t))]$ vs. $\ln (t)$.

the tail of KM, which is similarly not followed by both functions. The universal WF idea offers regression fit to the KM for a group of patients who have had an event or have censored until the end of the study. This is, of course, limited in real trials. We consider any chosen cohorts inhomogeneous because of the huge variability of living conditions. A homogenous group of patients, which has identical individuals could never be selected. However, there is a possibility to divide the cohort to subgroups with very similar patients, and fit WF on these independently, while the measured $\mathrm{KM}$ is, of course, a sum of the results of all the subgroups. With $\mathrm{M}$ subgroups in the complete cohort of $N$ patients, and every group containing $k_{1}, k_{2}, \cdots, k_{M}$ patients, the WF for the actual measured non-parametric KM will be:

$$
\begin{aligned}
& W^{(K M)}(t)=\frac{k_{1}}{N} \mathrm{e}^{-\left(\frac{t}{t_{0}^{(1)}}\right)^{n^{(1)}}}+\frac{k_{2}}{N} \mathrm{e}^{-\left(\frac{t}{t_{0}^{(2)}}\right)^{n^{(2)}}}+\cdots+\frac{k_{M}}{N} \mathrm{e}^{-\left(\frac{t}{t_{0}^{(M)}}\right)^{n^{(M)}}} \\
& \text { or } W^{(K M)}(t)=\sum_{i=1}^{M} \frac{k_{i}}{N} \mathrm{e}^{-\left(\frac{t}{t_{0}^{(i)}}\right)^{n^{(i)}}} \text { and } \sum_{i=1}^{M} k_{i}=N
\end{aligned}
$$


By taking extra care to have a homogeneous cohort, at least the time-limit of the study forms a group from patients, who had no event (or are not censored). The "remaining" patients in the given treatment study have the highest benefit from the performed treatment or they were in a definitely different condition when they were selected into the cohort. We call this group "remained group" (RG) due to the lack of proof of complete recovery. However, this group is sometimes regarded (incorrectly) as a cured fraction (according to the endpoints of the study). In a rigorous approach the disease-free survival (DFS) has to be compared with the matched healthy control group, and the cure-rate on this comparison must be decided [120]. An alternative way to determine the group of "cured" patients and the connected value of the "cure" time is when the hazard rate of the studied group corresponds to the hazard in the general population [121]. When it fits, we may talk about the real cure rate, which does not mean that an event cannot happen due to independent reasons from the investigated disease.

The KM curve in an RG situation obviously does not fit to the strict WF, which must be decreased to a zero cumulative probability. When the ratio of the remaining individuals is $c_{R G}=n^{R G} / N$, the KM plot can be approximated with reasonable accuracy by the weighted sum of two WFs. In the RG fraction, the time-parameter is longer than in the fraction of patients having an event or censored.

$$
W^{(c)}(t)=\left(1-c_{R G}\right) \mathrm{e}^{-\left(\frac{t}{t_{0}}\right)^{n}}+c_{R G} \mathrm{e}^{-\left(\frac{t}{t_{0}^{(R G)}}\right)^{n^{(R G)}}}
$$

In this case, the composition of the time-parameter of the long survival WF fit is practically infinite (compared to the time-length of the study):

$$
W^{(R G)}(t)=\mathrm{e}^{-\left(\frac{t}{t_{0}^{(R G)}}\right)^{n^{(R G)}}} \cong 1
$$

In this case, the correction by a survived fraction of the patients is constant. Denoting the constant correction $c$, the plot will be composed by this:

$$
W^{(c)}(t)=(1-c) \mathrm{e}^{-\left(\frac{t}{t_{0}^{(c)}}\right)^{n}}+c
$$

The variation of $\mathrm{c}$ shows different fitting functions, Figure 9:

Characterization of the curative effect of the treatment making a WF fit to the non-parametric KM survival could be done with the Shannon-entropy. Entropy measures the information carried by the probable density function (pdf, $\left.p\left(t, n, t_{0}\right)\right)$ behind the WF $\left(W\left(t, n, t_{0}\right)\right)$. It measures the probability of realization of an event or censoring

$$
\begin{aligned}
& p\left(t, n, t_{0}\right)=\frac{\mathrm{d} W\left(t, n, t_{0}\right)}{\mathrm{d} t}=\frac{n}{t_{0}}\left(\frac{t}{t_{0}}\right)^{n-1} \exp \left(-\left(\frac{t}{t_{0}}\right)^{n}\right) \\
& \int_{0}^{\infty} p\left(t, n, t_{0}\right) \mathrm{d} t=1
\end{aligned}
$$




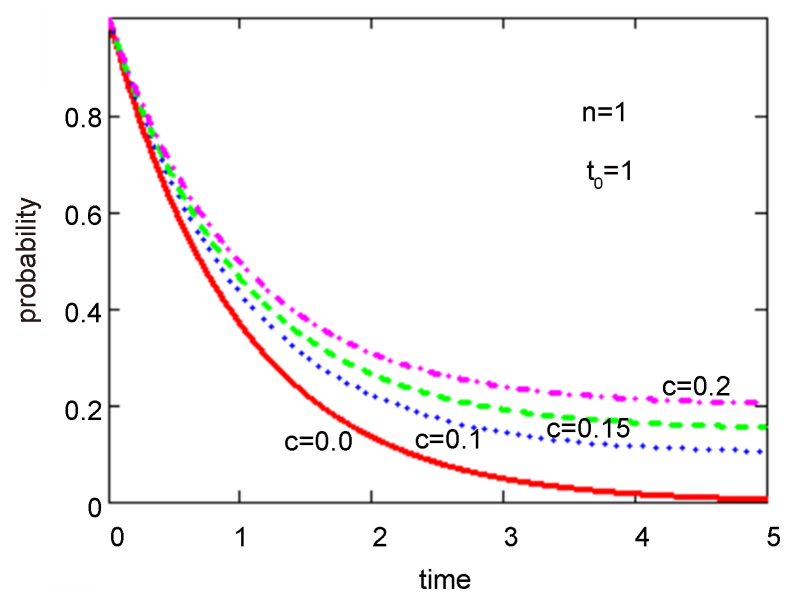

(a)

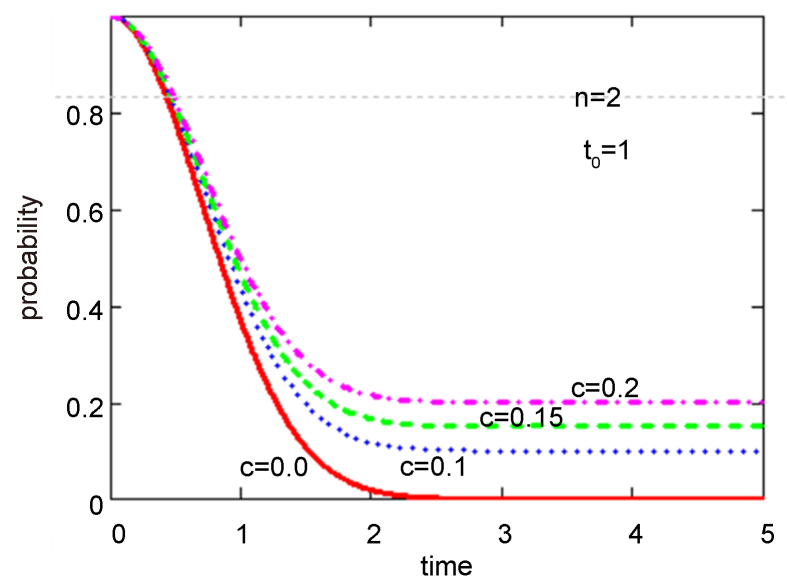

(b)

Figure 9. The WF with various $\mathrm{c}$ concentration of the patients in the RG group.

The quantity of information is $I\left(t, n, t_{0}\right)=-\ln \left(p\left(t, n, t_{0}\right)\right)$ which is realized by $p\left(t, n, t_{0}\right)$, so the complete information from the system is the classical Shannon-entropy, [122] is:

$$
S_{S h}\left(n, t_{0}\right)=-\int_{0}^{\infty} p\left(t, n, t_{0}\right) \ln \left(p\left(t, n, t_{0}\right)\right) \mathrm{d} t
$$

A higher entropy shows less information (more uncertainty). When an event has a lower probability to occur, it carries more information, so its Shannonentropy is lower than the effects of the frequent occurrence. The expectation of a random variable is characterized by this entropy, so by this meaning it is a direct analog for the entropy definition in physics (statistical thermodynamics). When the informational entropy decreases, (its change becomes negative) it means that the probability distribution differs from the uniformed distribution, concentrating to some data.

The entropy growth in physics usually happens when the system approaches equilibrium, while in pdf the increase of entropy shows a lack of information when the average rate of information produced by the stochastic source of the 
data decreases.

The Shannon entropy (28) measures the diversity of probability distribution function (pdf) behind WF (in fact the derivative of WF). It is a sum of the $n$ and $t_{0}$ dependent parts:

$$
\begin{aligned}
& S_{S h}\left(n, t_{0}\right)=\gamma\left(1-\frac{1}{n}\right)+\ln \left(\frac{t_{0}}{n}\right)+1=S_{S h 1}(n)+S_{S h 2}\left(t_{0}\right) \\
& \text { where } S_{S h 1}(n)=\gamma\left(1-\frac{1}{n}\right)-\ln (n)+1 ; S_{S h 2}\left(t_{0}\right)=\ln \left(t_{0}\right)
\end{aligned}
$$

and $\gamma$ is the Euler-Mascheroni constant: $\gamma \cong 0.577$ The special points of this entropy function are:

$$
\begin{aligned}
& S_{S h 2}(1)=0 ; \max \left(S_{S h 1}(n)\right)=S_{S h 1}(\gamma) \approx 1.127 \\
& S_{S h 1}(0.173) \cong 0 ; S_{S h 1}(4.223) \cong 0 ; S_{S h 1}(0.363) \cong 1 ; S_{S h 1}(1)=1 \\
& \lim _{n \rightarrow \infty, t_{0} \rightarrow \infty} S_{S h}\left(n, t_{0}\right)=-(1+\gamma)
\end{aligned}
$$

The entropy (diversity) monotonically grows by $t_{0}$ in a logarithmic way, while it rapidly grows by $n$ reaching the maximum at $n=\gamma \quad$ (when $t_{0}=1$ ) and decreases from that point reaching zero at $n=4.223$ (when $t_{0}=1$ ) and building information from that point (decreasing), so the step-function of WF (definite step) starts to dominate. The division of the entropy of a shape and scale (time) dependent part gives a possibility to define the role of these parameters. While the scale (time) parameter increases the Shannon-entropy monotonically, the shape parameter $(n)$ after a maximum at $\gamma$, decreases the entropy, showing an increasing amount of information about the death (decreasing info about being alive) of the participants in the cohort. The growing shape-factor $n$ definitely worsens the survival over the value $\gamma$, while the growth of the scale (time) factor gives longer survival expectations.

The Shannon entropy could be calculated real-time $t\left(S_{S h}\left(n, t_{0}\right)\right)$ and also could be relative to $t_{0}$ time, meaning, that the time is measured in $t_{0}$ units $\left(S_{S h 0}(n)=S_{S h}(n, 1)\right)$, estimating the self-time. A higher entropy value means a higher uncertainty of death (therefore, a lower certainty of being alive). We expectthe growth of Shannon entropy of the parametric probability distribution function in cases of better results of the treatment.

\section{Discussion}

To demonstrate the parametrization, we use a large number of patients (1180 individuals), with various tumors treated by numerous standard therapies, but having one thing in common: they are treated by complementary modulated electro-hyperthermia (mEHT), when the standard treatment fail to deliver the desirable results, [123] [124]; Figure 10.

Using the approximate parametrization by the evaluation of this KM plot with the slope in $t_{0}$, we get $t_{0} \approx 43$ and $n \approx 0.9$. median $\approx 28$, Figure 11 .

The fit of single parametric WF curve to the KM plot, (Figure 12). The single 

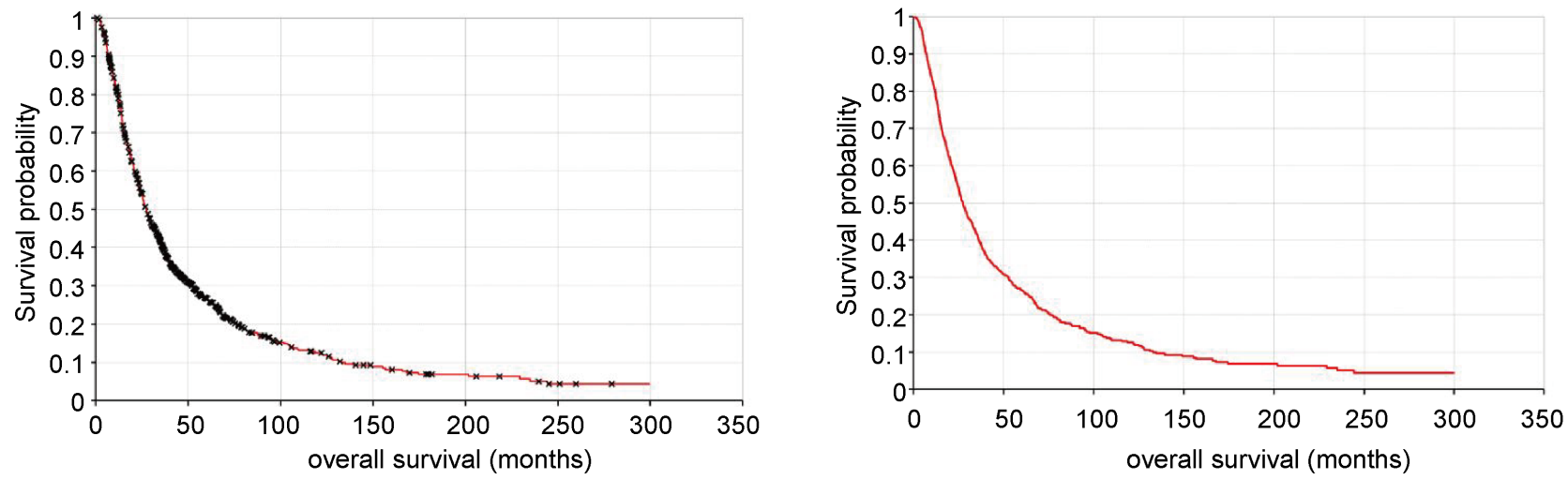

Figure 10. The overall survival KM-plot of a large number of patients (Pts.) = 1180 patients (various advanced solid malignancies, treated by complementary modulated electro-hyperthermia (mEHT), [117] [118]. The KM-plot contains very long (25 y) survival too. (a) with censored cases, (b) without censoring (for clarity).

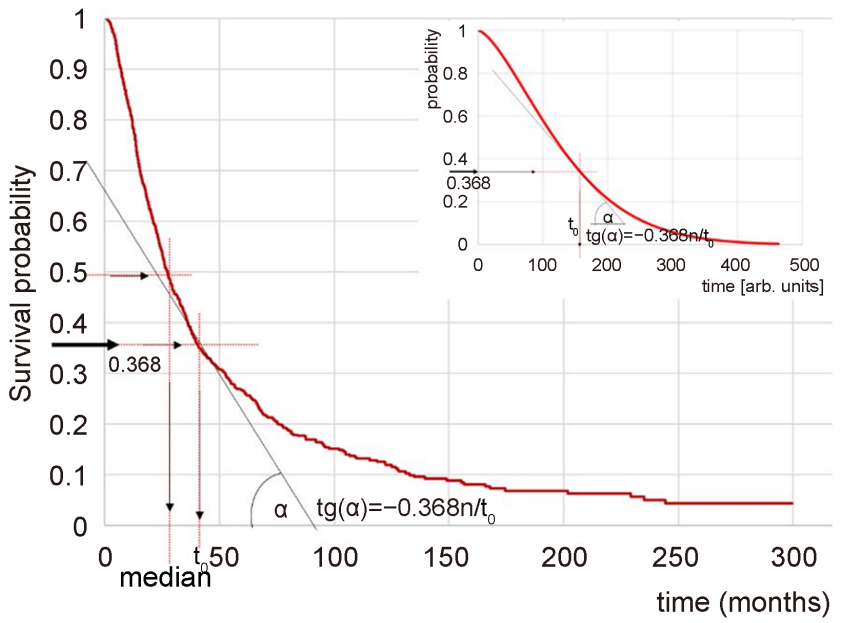

Figure 11. Rough determination of the parameters of the Weibull-fit to KM. Real process on a KM of Pts. $=1180$ patients suffering in various malignant diseases [117], Figure 10. The obtained parameters are: $t_{0} \approx 43$ and $\operatorname{tg}(\alpha) \approx 0.7 / 85 \approx 0.008$, hence $n \approx 0.9$. Control: median $\approx 28$, which is approximately correct. (The principle of the process is in the insert in the figure).

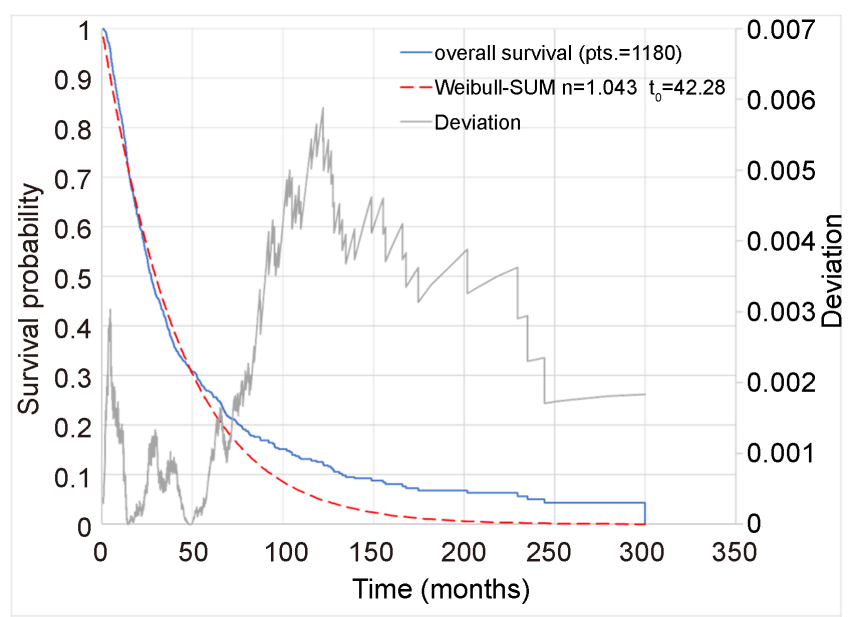

(a) 


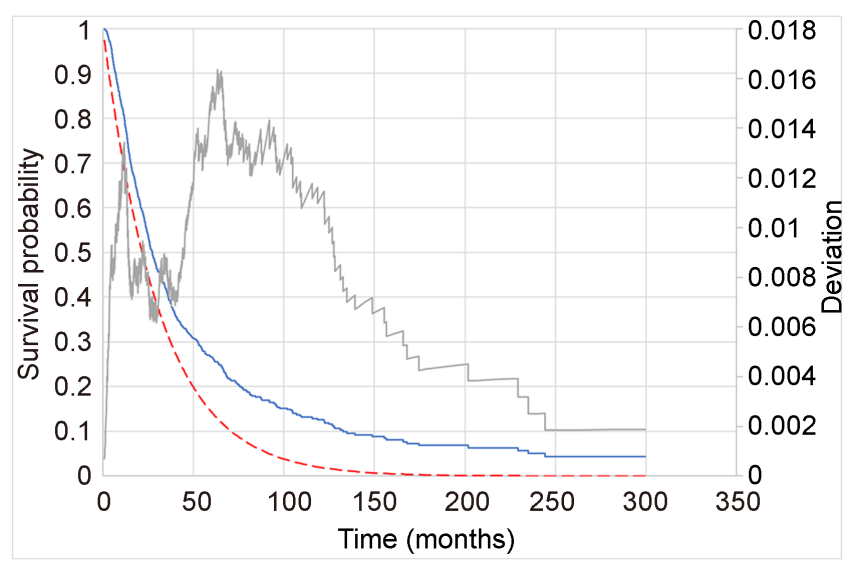

(b)

Figure 12. WF fit (dashed line) to KM solid line to the overall survival KM-plot of pts. $=1180$ patients (Figure 10) (a) Regression by deviation minimum $\mathrm{SE}=1.6544 ; r^{2}=0.9850, n=1.043 ; t_{0}=42.28$; $S_{S h}=4.726$; (b) regression by correlation maximum. $\mathrm{SE}=15.422$; $r^{2}=0.9969, n=1.013, t_{0}=31.142 ; S_{S h}=4.433$.

WF fits with an acceptable accuracy; the largest deviation is less than 0.007, $(0.7 \%)$.

Note, that there is a difference, when we fit by minimalizing the deviation of the curves, $\mathrm{SE}=\min \left\{\sum_{i}\left(x_{i}^{K M}-x_{i}^{W F}\right)^{2}\right\}$ or

$r^{2}=\left(\frac{\sum_{i}\left(x_{i}^{K M}-\left\langle x^{K M}\right\rangle\right)\left(x_{i}^{W F}-\left\langle x^{W F}\right\rangle\right)}{\sqrt{\sum_{i}\left(x_{i}^{K M}-\left\langle x^{K M}\right\rangle\right)} \sqrt{\sum_{i}\left(x_{i}^{W F}-\left\langle x^{W F}\right\rangle\right)}}\right)^{2}$ the square of Pearson correlation (where the \langle\rangle bracket means the mean of the variable). The obvious difference is due to the different meaning of fit. The parameter SE minimizes the difference between the curves, while the $r^{2}$ minimizes the shape difference (maximizes the similarities) of the curves. A comparison with Shannon entropy shows more certainty (less uncertainty) by about $6 \%$ in the regression by minimizing SE than maximizing $r^{2}$. In the following, when we do not note the opposite, we use the minimal SE regression.

The fit is accurate, having no more difference in any compared points of the curves than 1\%, but it is not accurate enough at the end of the observed time, due to the RG group of the patients. The deviation could be less with applying the RG principle of (26), Figure 13. The $S_{S h}=4.543$, which is $2.5 \%$ higher, mirrors the RG part of the patient distribution.

The parametric decomposition gives better fit by two WFs according to (24), Figure 14, where the $r^{2}$ has reduced drastically. The result shows the responding group (response rate (RR) 48\%) and the non-responding one (52\%). Note, that the less-responding group could be regarded as a non-responding control-arm.

The long-survival part of KM-plot has a higher entropy and shows more uncertainty of the death in both approaches. A better fit can be achieved when we 


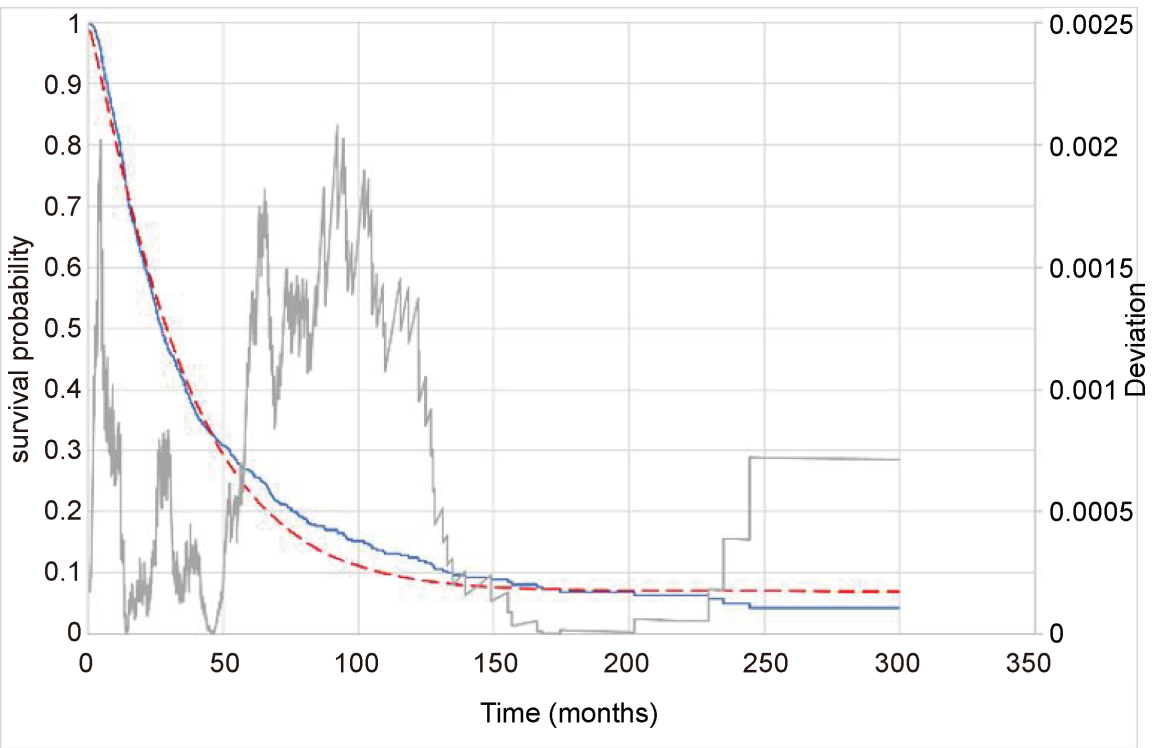

Figure 13. WF fit (dashed line) to KM solid line) from Figure 10. The applied RG is 7\%. $\mathrm{SE}=0.9059 ; r^{2}=0.9918, n=1.135, t_{0}=36.632 ; S_{S h}=4.543$. The largest square of deviation of the point-pairs (LD) is $0.002(0.2 \%)$.

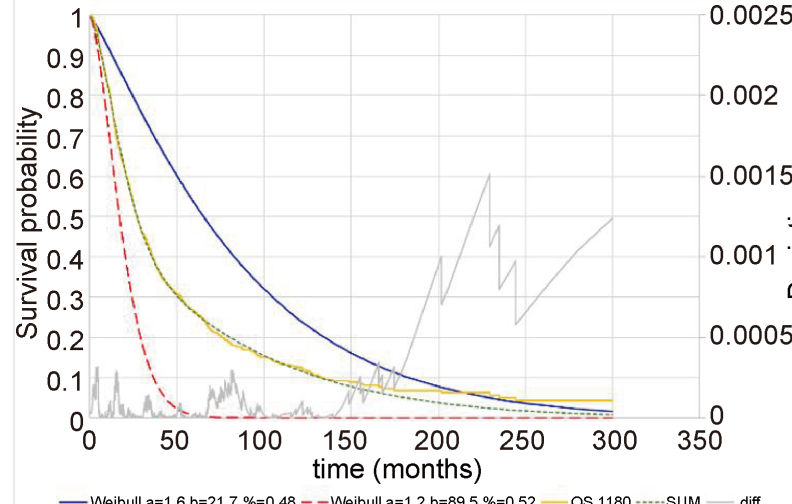

(a)

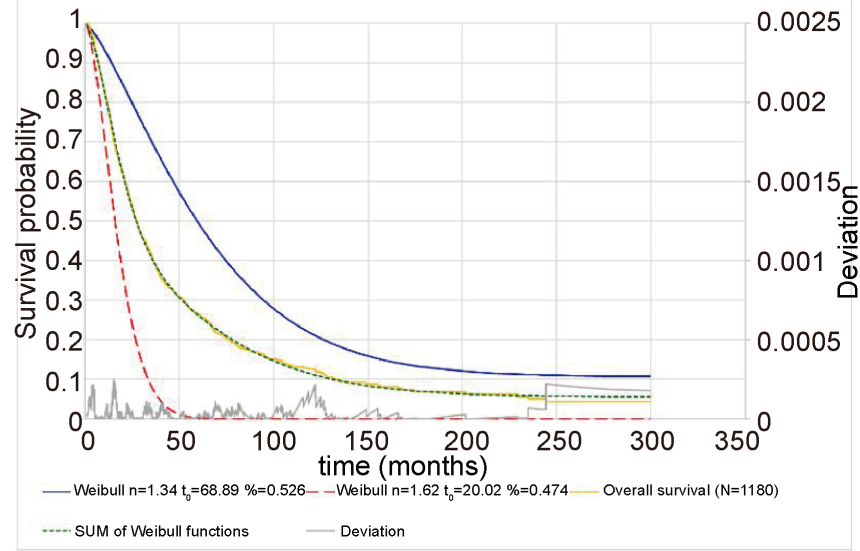

(b)

Figure 14. (a) The double Weibull fit to the overall survival of $n=1180$ patients in malignant diseases complementary treated by the mEHT method. The longer survival (solid line) is the group of responding patients for the treatment; while the shorter survival time (dashed line) is a component of the composite fit of WF regarded as a non-responding group, that could be used as a reference cohort of patients. The sum of the two components (dotted line) fits to the measured overall survival. Deviation of the regression is shown in light solid line with values on the secondary axes. (a) Decomposition using both WF without RG ( $R G=0$, $\left.\mathrm{RR}=48.4 \%, \mathrm{SE}=0.0968 ; r^{2}=0.99954\right)$; longer survival component $\left(n=1.17, t_{0}=89.52 ; S_{S h}=5.423\right)$; shorter survival component ( $\left.n=1.59, t_{0}=21.74 ; S_{S h}=3.831\right)$; (b) Decomposition using RG ( RG=10.7\%, RR =52.6\%, SE =0.0596 ;

$\left.r^{2}=0.99954\right)$; longer survival component $\left(n=1.34, t_{0}=68.89 ; S_{S h}=5.086\right)$; shorter survival component ( $n=1.62$, $\left.t_{0}=20.02 ; S_{S h}=3.734\right)$. KM-plot and the sum of decomposed Weibull curves suppressed are remarkable (solid line) compare to the single fit (dotted line).

count RG. The RG is obtained from the remaining survival fraction in most of the actual cases, and it has measurably longer survival than the study follows the patients who had no event or were not censored earlier. RG is a part of the 
"censored" patients at the end of the study.

For an easier calculation of the WF fractions (components) of the KM-plot, we may use the logarithmic evaluation of the survivals, which modifies the grouping more than the above decomposition. A linearly fit function $\ln [-\ln (W(t))]$ by $\ln (t)$ of KM is shown in Figure 10. According to (22) it shows rather large deviations at the start and at the end of the curve, Figure 15.

The original WF fit shown in Figure 12(a), and the linear fit from the logarithmic approach of Figure 15. differs from each other, Figure 16. The deviation of the logarithmic fit is more than double in some intervals, so the direct fit of WF to KM is more accurate.

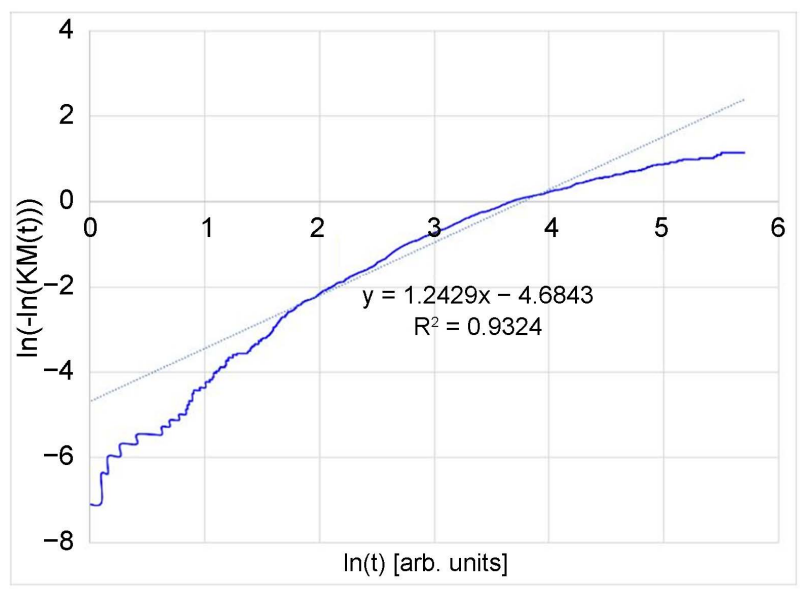

Figure 15. The logarithmic fits of WF to KM of Figure 10. The linear fit to the complete curve gives two parameters: $\mathrm{SE}=1.6544$; $r^{2}=0.9850, n=1.2429 ; t_{0}=43.33 ; S_{S h}=4.664$.

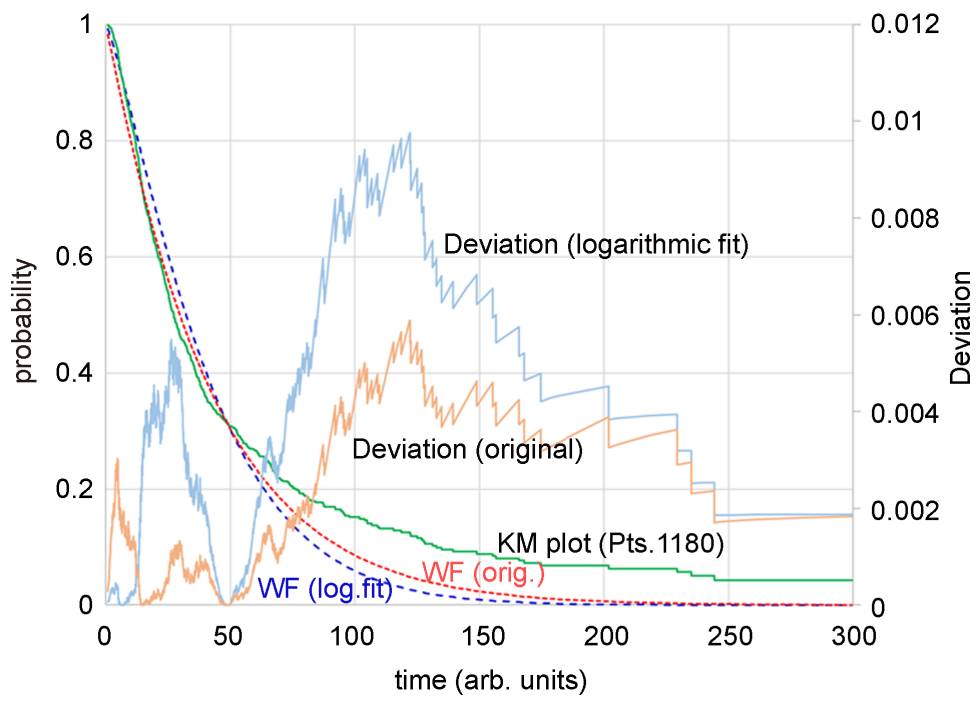

Figure 16. The logarithmic fits of WF to KM of Figure 10. The linear fit to the complete curve gives two parameters: $\mathrm{SE}=3.93 ; n=1.2429 ; t_{0}=43.33 ; S_{S h}=4.664$. The deviation from the parameters of the original fit $\left(\mathrm{SE}=1.6544 ; r^{2}=0.9850, n=1.043\right.$; $t_{0}=42.28 ; S_{S h}=4.726$ ) shown in Figure 12(a). 
Despite the inaccuracy of the logarithmic evaluation, it has a great advantage of guessing the subgroups of the patients by an optimal decomposing of the KM plot. The logarithmic curve on Figure 15. shows three well distinguishable parts, for which the linear is accurate, and divides the original KM into three subgroups, Figure 17.

The WF fit to Figure 17 of the three parts of the KM is shown on Figure 18.

The logarithmic fit by (22) shows different results than the direct fit. The reason is simply that the logarithmic fit considers only a part of the whole curve, and fits to that, consequently the accurate fit to that part of the KM will not fit to the other parts at all, if the logarithmic curve was approached in different parts. The observed KM is, of course, considers all the patients. The overlapping fits from the logarithmic approach modifies the KM plot. Consequently, only the fit for original KM plot has a relevance.

However, the logarithmic analysis is very useful for detecting the subgroups of the patients. It became clear that the survival contains three subgroups, Figure 17. Consequently, three partitions of the KM curve (Figure 10) would give a
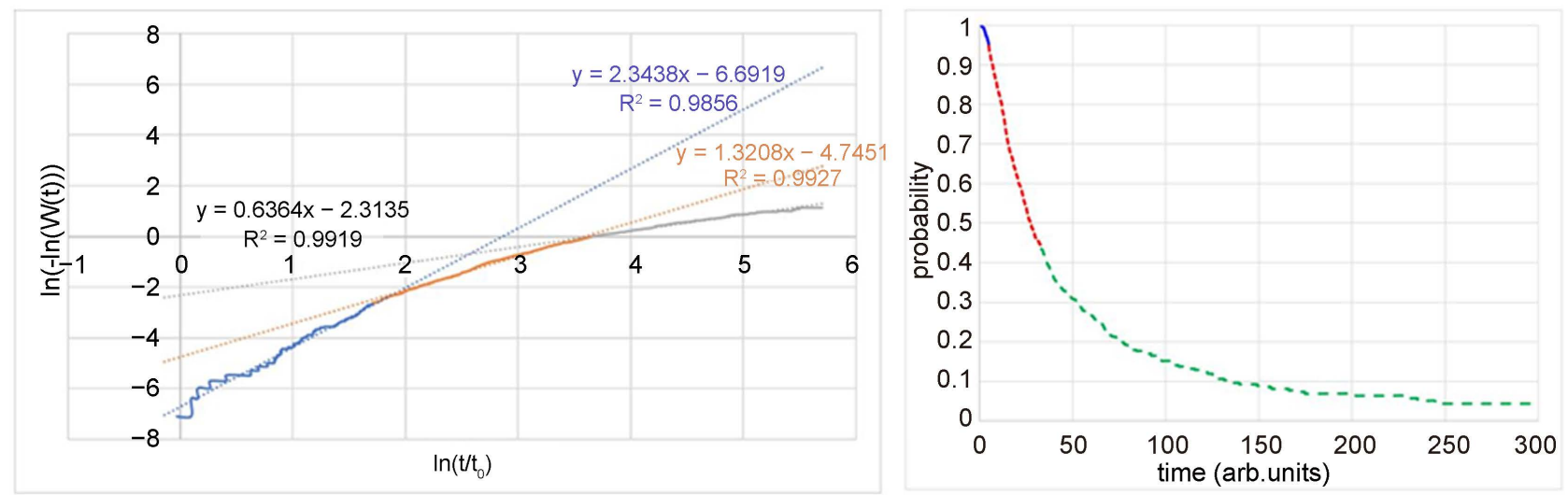

Figure 17. The various logarithmic fits to $\mathrm{KM}$ of Figure 10. (a) Linear fit to three fraction of the KM curve, $r_{1}^{2}=0.9856$, $r_{2}^{2}=0.9927, r_{3}^{2}=0.9919$, (b) Using the linear fits, the original curve may be fractioned to the three subgroups, (solid, dots and dashed lines).
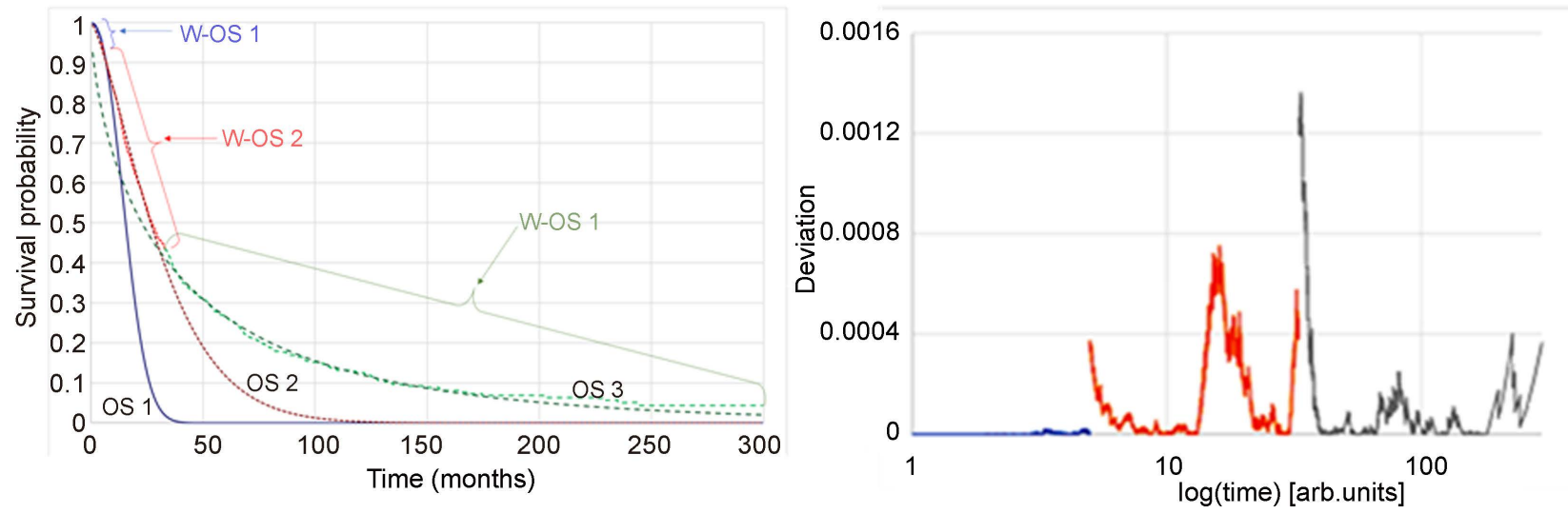

Figure 18. Fitting the KM by WFs according to the logarithmic fit on Figure 17. (a) curves, $n_{1}=2.344, t_{01}=17.347 ; S_{S h 1}=$ $3.332 ; n_{2}=1.321, t_{02}=36.328 ; S_{S h 2}=4.454 ; n_{3}=0.6364, t_{03}=37.913 ; S_{S h 3}=4.758$. (b) deviations by groups. 


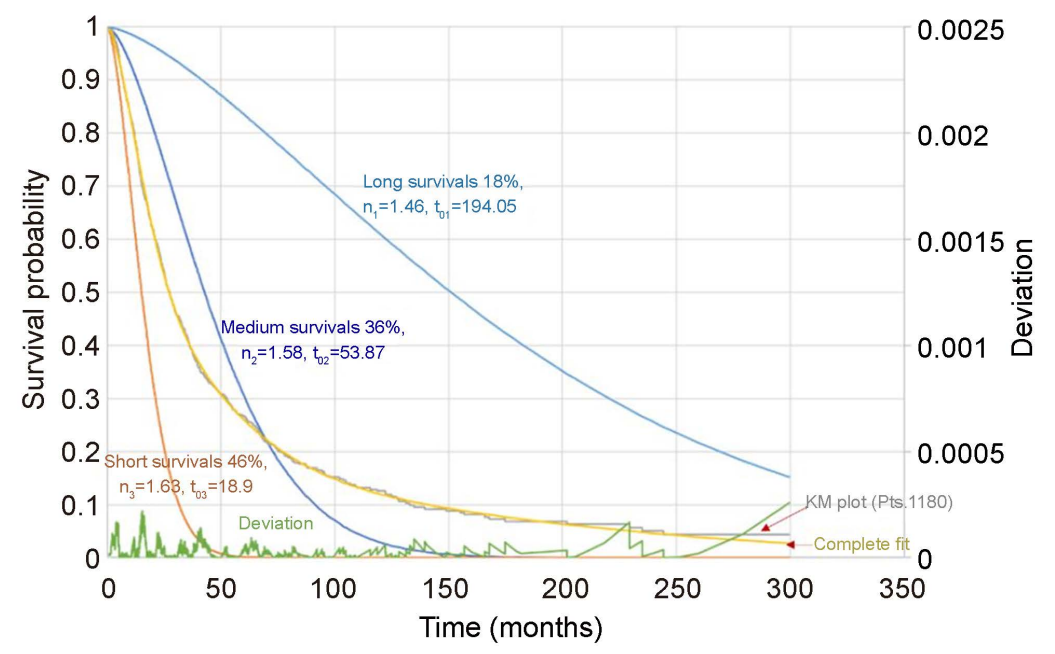

Figure 19. Decomposition of the KM on Figure 10. (Pts. $=1180)$ to three groups (long 18\%: $n_{1}=1.46, n_{1}=1.46$, median $_{1}=151.02, S_{S h 1}=6.071 ;$ medium (36\%): $n_{2}=1.58, t_{02}=53.87, \operatorname{median}_{2}=42.73, S_{S h 2}=4.741 ;$ short $(46 \%): n_{3}=1.63$, $t_{03}=18.9, \operatorname{median}_{3}=15.09, S_{S h 3}=3.674$. The deviation of the fit remains under $0.0005(0.05 \%)$.

more accurate fit, than the RG (Figure 13) or the two-group decomposition (Figure 14) had allowed. This fit to KM is very accurate, the deviation remains under 0.0005 in the complete fit, Figure 19.

\section{Conclusions}

We had shown the applicability of the two-parameter cumulative Weibull distribution for approximating the non-parametric Kaplan-Meier plot with a higher accuracy. We had shown the universality of the Weibull approach based on the general behaviors of the living organisms, including the cancer-tissue development. The self-organizing and self-similarity with their consequences determine the strict connection of the parametric approach well with the experimental non-parametric observations. Informational entropy allows the distinguishing of the subgroups in a general set of patients by their overall survival.

We have demonstrated that applying the two-parameter WF provides a sufficient fit to the non-parametric KM survival curve in a real case of 1180 patients suffering in various malignant diseases. Two of the 3 characteristic parameters of the KM plot (namely the points of median, mean or inflection) are enough to reconstruct the parametric fit.

In summary, Weibull parametric distribution with satisfactory refinement can accurately approximate a KM survival plot with surviving individuals at the endpoint of the study.

\section{Acknowledgements}

This work was supported by the Hungarian Competitiveness and Excellence Programme grant (NVKP_16-1-2016-0042). 


\section{Conflicts of Interest}

The authors declare no conflicts of interest regarding the publication of this paper.

\section{References}

[1] Szentgyorgyi, A. (2019) Life Is Nothing But an Electron Looking for a Place to Rest. https://www.goodreads.com/work/quotes/46106688-the-big-picture-on-the-originsof-life-meaning-and-the-universe-itsel

[2] Walleczek, J. (2000) Self-Organized Biological Dynamics \& Nonlinear Control. Cambridge Univ. Press, Cambridge. https://doi.org/10.1017/CBO9780511535338

[3] Kauffman, S.A. (1993) The Origins of Order: Self-Organization and Selection in Evolution. Oxford University Press, New York, Oxford. https://doi.org/10.1007/978-94-015-8054-0_8

[4] Haken, H. (1987) Self-Organization and Information. Physica Scripta, 35, 247-254. https://doi.org/10.1088/0031-8949/35/3/006

[5] Sornette, D. (2000) Chaos, Fractals, Self-Organization and Disorder: Concepts and Tools. Springer Verlag, Berlin, Los Angeles.

[6] Wolfram, S. (2002) A New Kind of Science. Wolfram Media Inc., Champaign.

[7] Camazine, S., Deneubourg, J.L., Franks, N.R., et al. (2003) Self-Organization in Biological Systems. Princeton Studies in Complexity, Princeton Univ. Press, Princeton, Oxford.

[8] Bozorgmehr, J.E.H. (2014) The Role of Self-Organization in Developmental Evolution. Theory in Biosciences, 133, 145-163.

https://doi.org/10.1007/s12064-014-0200-4

[9] Johnson, B.R. and Lam, S.K. (2010) Self-Organization, Natural Selection, and Evolution: Cellular Hardware and Genetic Software. Bioscience, 60, 879-885. https://doi.org/10.1525/bio.2010.60.11.4

[10] Glancy, J., Sone, J.V. and Wilson, S.P. (2016) How Self-Organization Can Guide Evolution. Royal Society Open Science, 3, Article ID: 160553. https://doi.org/10.1098/rsos.160553

[11] Kurakin, A. (2011) The Self-Organizing Fractal Theory as a Universal Discovery Method: The Phenomenon of Life. Theoretical Biology and Medical Modelling, 8, 4. https://doi.org/10.1186/1742-4682-8-4

[12] Scheffer, M. and Nes, V.E.H. (2006) Self-Organized Similarity, the Evolutionary Emergence of Groups of Similar Species. PNAS, 103, 6230-6235. https://doi.org/10.1073/pnas.0508024103

[13] Deering, W. and West, B.J. (1992) Fractal Physiology. IEEE Engineering in Medicine and Biology, 11, 40-46. https://doi.org/10.1109/51.139035

[14] West, B.J. (1990) Fractal Physiology and Chaos in Medicine. World Scientific, Singapore, London.

[15] Bassingthwaighte, J.B., Leibovitch, L.S. and West, B.J. (1994) Fractal Physiology. Oxford Univ. Press, New York, Oxford. https://doi.org/10.1007/978-1-4614-7572-9

[16] Li, W. (1989) Spatial 1/f Spectra in Open Dynamical Systems. Europhysics Letters, 10, 395-400. https://doi.org/10.1209/0295-5075/10/5/001

[17] Schlesinger, M.S. (1987) Fractal Time and 1/f Noise in Complex Systems. Annals of the New York Academy of Sciences, 504, 214-228. https://doi.org/10.1111/j.1749-6632.1987.tb48734.x 
[18] Bak, P., Tang, C. and Wieserfeld, K. (1988) Self-Organized Criticality. Physical Review $A$, 38, 364. https://doi.org/10.1103/PhysRevA.38.364

[19] Musha, T. and Sawada, Y. (1994) Physics of the Living State. IOS Press, Amsterdam.

[20] von Bertalanffy, L. and Pirozynski, W.J. (1953) Tissue Respiration, Growth, and Basal Metabolism. Biology Bulletin, 105, 240-256. https://doi.org/10.2307/1538640

[21] von Bertalanffy, L. (1968) General System Theory: Foundations, Development, Applications. George Braziller, New York.

[22] Labra, F.A., Marquet, P.A. and Bozinovic, F. (2007) Scaling Metabolic Rate Fluctuations. PNAS, 104, 10900-10903. https://doi.org/10.1073/pnas.0704108104

[23] Goldberger, A.L., Amaral, L.A., Hausdorff, J.M., et al. (2002) Fractal Dynamics in Physiology: Alterations with Disease and Aging. PNAS Colloquium, 99, 2466-2472. https://doi.org/10.1073/pnas.012579499

[24] Szendro, P., Vincze, G. and Szasz, A. (2001) Pink Noise Behaviour of the Bio-Systems. European Biophysics Journal, 30, 227-231. https://doi.org/10.1007/s002490100143

[25] Calder III, W.A. (1984) Size, Function and Life History. Dover Publications Inc., Mineola, New York.

[26] West, G.B. and Born, J.H. (2000) Scaling in Biology. Oxford University Press, Oxford.

[27] West, G.B., Woodruf, W.H. and Born, J.H. (2002) Allometric Scaling of Metabolic Rate from Molecules and Mitochondria to Cells and Mammals. Proceedings of the National Academy of Sciences of the United States of America, 99, 2473-2478. https://doi.org/10.1073/pnas.012579799

[28] West, G.B. and Brown, J.H. (2005) The Origin of Allometric Scaling Laws in Biology from Genomes to Ecosystems: Towards a Quantitative Unifying Theory of Biological Structure and Organization. Journal of Experimental Biology, 208, 15751592. https://doi.org/10.1242/jeb.01589

[29] Lane, N. (2006) Mitochondria: Key to Complexity. In: Martin, W., Ed., Origins of Mitochondria and Hydrogenosomes, Springer, Heidelberg, Chapter 2. https://doi.org/10.1007/978-3-540-38502-8_2

[30] Brown, J.H., West, G.B. and Enquis, B.J. (2005) Yes, West, Brown and Enquist's Model of Allometric Scaling Is Both Mathematically Correct and Biologically Relevant. Functional Ecology, 19, 735-738. https://doi.org/10.1111/j.1365-2435.2005.01022.x

[31] Glazier, D.S. (2014) Metabolic Scaling in Complex Living Systems. Systems, 2, 451540. https://doi.org/10.3390/systems2040451

[32] Kolmogorov, N.N. (1937) On the Statistical Theory of the Crystallization of Metals. Bull Acad Sci UssR Math Ser, 1, 355-359.

[33] Joshnson, W.A. and Mehl, P.A. (1939) Reaction Kinetics in Processes of Nucleation and Growth. Transactions of the American Institute of Mining, Metallurgical and Petroleum Engineers, 135, 416.

[34] Avrami, M.A. (1939) Kinetics of Phase Change, Parts: I-II-III. The Journal of Chemical Physics, 7, 1103.

[35] Levine, L.E., Narayan, K.L. and Kelton, K.F. (1997) Finite Size Corrections for the Johnson-Mehl-Avrami-Kolmogorov Equation. Journal of Materials Research, 12, 124-131. https://doi.org/10.1557/JMR.1997.0020

[36] Fanfoni, M., Persichetti, L. and Tomellini, M. (2012) Order and Randomness in Kolmogorov-Johnson-Mehl-Avrami-Type Phase Transitions. Journal of Physics. Condensed Matter, 24, Article ID: 355002. 
https://doi.org/10.1088/0953-8984/24/35/355002

[37] Cope, F.W. (1977) Detection of Phase Transitions and Cooperative Interactions by Avrami Analysis of Sigmoid Biological Time Curves for Muscle, Nerve, Growth, Firefly, and Infrared Phosphorescence, of Green Leaves, Melanin and Cytochrome C. Physiological Chemistry and Physics, 9, 443-459.

[38] Cope, F.W. (1977) Solid State Physical Replacement of Hodgkin-Huxley Theory. Phase Transformation Kinetics of Axonal Potassium Conductance. Physiological Chemistry and Physics, 9, 155-160.

[39] Zhang, H. (2005) Reconstructing DNA Replication Kinetics from Small DNA Fragments. Ms. Thesis, Simon Fraser University, Burnaby.

https://doi.org/10.1103/PhysRevE.73.051903

[40] Cope, F.W. (1977) The Kinetics of Biological Phase Transitions Manifested by Sigmoid Time Curves: A Review of Approaches. Physiological Chemistry and Physics, 8, 519-527.

[41] Cope, F.W. (1980) Avrami Analysis of Electrical Switching in Hydrated Melanin Suggest Dependence on a Phase Transition. Physiological Chemistry and Physics, 12, 537-538.

[42] Suckjoon, J. and Bechhoefer, J. (2005) Nucleation and Growth in One Dimension. II. Application to DNA Replication Kinetics. Physical Review E, 71, Article ID: 011909. https://doi.org/10.1103/PhysRevE.71.011909

[43] Zhang, H.Y. (2005) Reconstructing DNA Replication Kinetics from Small DNA Fragments. Simon Fraser University, Burnaby.

[44] Szasz, O., Szigeti, G.P. and Szasz, A. (2017) On the Self-Similarity in Biological Processes. Open Journal of Biophysics, 7, 183-196.

https://doi.org/10.4236/ojbiphy.2017.74014

[45] Binney, J.J., Dowrick, N.J., Fisher, A.J., et al. (1992) The Theory of Critical Phenomena, an Introduction to the Renormalization Group. Oxford Science Publications, Oxford.

[46] Augis, J.A. and Bennett, J.E. (1978) Calculation of the Avrami Parameters for Heterogeneous Solid State Reactions Using a Modification of the Kissinger Method. Journal of Thermal Analysis, 13, 285-291. https://doi.org/10.1007/BF01912301

[47] Jena, A.K. and Chaturvedi, M.C. (1992) Phase Transformations in Materials. Prentice Hall, Upper Saddle River, 247.

[48] Aloev, V.Z., Kozlov, G.V. and Zaikov, G.E. (2004) Relationship between the Exponent of the Kolmogorov-Avrami Equation and the Fractal Dimension in the Crystallisation of Uniaxially Stretched Crosslinked Polychloroprene. Kauchuk I Rezina, No. 3, 38-39. https://doi.org/10.1177/0307174X0403101210

[49] Hassan, M.K. and Kurths, J. (2004) Scale Invariant Fractal and Slow Dynamics in Nucleation and Growth Processes. https://arxiv.org/pdf/cond-mat/0407715.pdf

[50] Cope, F.W. (1977) Detection of Phase Transitions and Cooperative Interactions by Avrami Analysis of Sigmoid Biological Time Curves for Muscle, Nerve, Growth, Firefly, and Infrared Phosphorescence, of Green Leaves, Melanin and Cytochrome C. Physiological Chemistry and Physics, 9, 443-459.

[51] Cope, F.W. (1977) Solid State Physical Replacement of Hodgkin-Huxley Theory. Phase Transformation Kinetics of Axonal Potassium Conductance. Physiological Chemistry and Physics, 9, 155-160.

[52] Cope, F.W. (1980) Avrami Analysis of Electrical Switching in Hydrated Melanin Suggest Dependence on a Phase Transition. Physiological Chemistry and Physics, 
$12,537-538$.

[53] Izquierdo-Kulich, E. and Nieto-Villar, J.M. (2013) Morphogenesis and Complexity of the Tumor Patterns. In: Rubio, R.G., Ryazantsev, Y.S., Starov, V.M., Huang, G.-X., Chetverikov, A.P., Arena, P., Nepomnyashchy, A.A., Ferrus, A. and Morozov, E.G., Eds., Without Bounds. A Scientific Canvas of Nonlinearity and Complex Dynamics. Understanding Complex Systems, Springer-Verlag, Berlin Heidelberg, 657-691. https://doi.org/10.1007/978-3-642-34070-3_48

[54] Davies, P.C.W., Demetrius, L. and Tuszynski, J.A. (2011) Cancer as a Dynamical Phase Transition. Theoretical Biology and Medical Modelling, 8, 30. https://doi.org/10.1186/1742-4682-8-30

[55] Enderling, H., Hahnfeldt, P., Hlatky, L. and Almog, N. (2012) Systems Biology of Tumor Dormancy: Linking Biology and Mathematics on Multiple Scales to Improve Cancer Therapy. Cancer Research, 72, 2172-2175. https://doi.org/10.1158/0008-5472.CAN-11-3269

[56] Szasz, O., Szigeti, G.P., Szasz, A. and Benyo, Z. (2018) Role of Electrical Forces in Angiogenesis. Open Journal of Biophysics, 8, 49-67.

https://doi.org/10.4236/ojbiphy.2018.82005

[57] Szasz, O., Vincze, Gy., Szigeti, Gy.P., Benyo, Z. and Szasz, A. (2018) An Allometric Approach of Tumor-Angiogenesis. Medical Hypothesis, 116, 74-78. https://doi.org/10.1016/j.mehy.2018.03.015

[58] Szasz, O., Szigeti, G.P. and Szasz, A. (2018) The Intrinsic Self-Time of Biosystems. Open Journal of Biophysics, 9, 131-145.

[59] González, M.M., Joa, J.A.G., Cabrales, L.E.B., Pupo, A.E.B., Schneider, B., Kondakci, S., Ciria, H.M.C., Reyes, J.B., Jarque, M.V., O’Farril Mateus, M.A., Tamara Rubio González, T.R., Brooks, S.C.A., Cáceres, J.L.H. and González, G.V.S. (2017) Is Cancer a Pure Growth Curve or Does It Follow a Kinetics of Dynamical Structural Transformation? BMC Cancer, 17, 174. https://doi.org/10.1186/s12885-017-3159-y

[60] Izquierdo-Kulich, E.E., Alonso-Becerra and Nieto-Villar, J.M. (2011) Entropy Production Rate for Avascular Tumor Growth. Journal of Modern Physics, 2, 615. https://doi.org/10.4236/jmp.2011.226071

[61] Betancourt-Mar, J.A., Cocho, G., Mansilla, R. and Nieto-Villar, J.M. (2017) What Can Be Learned from a Phase Transitions in Tumor Growth? Insights in Biomedicine, 2,1 .

[62] Agus, D.B., Alexander, J.F., Arap, W., Ashili, S., Aslan, J.E., Austin, R.H., Backman, V., Bethel, K.J., et al. (2013) A Physical Sciences Network Characterization of Non-Tumorigenic and Metastatic Cells. Scientific Reports, 3, Article No. 1449. https://doi.org/10.1038/srep01449

[63] Stehlik, M., Mrkvicka, T., Filus, J. and Filus, I. (2012) Recent Developments on Testing in Cancer Risk: A Fractal and Stochastic Geometry. Journal of Reliability and Statistical Studies, 5, 83-95.

[64] Guiot, C., Degiorgis, P.G., Delsanto, P.P., Gabriele, P. and Deisboeck, T.S. (2003) Does Tumor Growth Follow a "Universal Law"? Journal of Theoretical Biology, 225, 147-151. https://doi.org/10.1016/S0022-5193(03)00221-2

[65] Bose, P., Brockton, N.T., Guggisberg, K., Nakoneshny, S.C., Kornaga, E., Klimowicz, A.C., Tambasco, M. and Dort, J.C. (2015) Fractal Analysis of Nuclear Histology Integrates Tumor and Stromal Features into a Single Prognostic Factor of the Oral Cancer Microenvironment. BMC Cancer, 15, 409. https://doi.org/10.1186/s12885-015-1380-0

[66] Sole, R.V. (2003) Phase Transitions in Unstable Cancer Cell Populations. The Euro- 
pean Physical Journal B, 35, 117-123. https://doi.org/10.1140/epjb/e2003-00262-8

[67] Loeb, L.A., Loeb, K.R. and Anderson, J.P. (2003) Multiple Mutations and Cancer. PNAS, 100, 776-781. https://doi.org/10.1073/pnas.0334858100

[68] Bielas, J.H., Loeb, K.R., Rubin, B.P., True, L.D. and Loeb, L.A. (2006) Human Cancers Express a Mutator Phenotype. PNAS, 103, 18238-18242. https://doi.org/10.1073/pnas.0607057103

[69] Neumann, J. (1928) Zur Theorie der Gesellschaftsspiele. Mathematische Annalen, 100, 295-320. (English Translation: Tucker, A.W. and Luce, R.D. (1959) On the Theory of Games of Strategy. Contributions to the Theory of Games, 4, 13-42. https://doi.org/10.1007/BF01448847

[70] Ben-David, S., Borodin, A., Karp, R., Tardos, G. and Wigderson, A. (1994) On the Power of Randomization in On-Line Algorithms. Algorithmica, 11, 2-14. https://doi.org/10.1007/BF01294260

[71] Si, T. (2008) Game Theory and Topological Phase Transition.

[72] Menon, S.N., Sasidevan, V. and Sinha, S. (2008) Emergence of Cooperation as a Non-Equilibrium Transition in Noisy Spatial Games. Frontiers in Physics, 6, 34. https://doi.org/10.3389/fphy.2018.00034

[73] Kaplan, E.L. and Meier, P. (1958) Nonparametric Estimation from Incomplete Observations. Journal of the American Statistical Association, 53, 457-481. https://doi.org/10.1080/01621459.1958.10501452

[74] Etikan, I., Abubakar, S. and Alkassim, R. (2017) The Kaplan Meier Estimate in Survival Analysis. Biometrics \& Biostatistics International Journal, 5, Article ID: 00128. https://doi.org/10.15406/bbij.2017.05.00128

[75] Efron, B. (1967) The Two-Sample Problem with Censored Data. Proceedings of the Fifth Berkeley Symposium on Mathematical Statistics and Probability, Vol. 4, 831852.

[76] Gill, R.D. (1980) Censoring and Stochastic Integrals. Mathematical Centre Tract No. 124, Mathematisch Centrum, Amsterdam. https://doi.org/10.1111/j.1467-9574.1980.tb00692.x

[77] Birnbaum, Z.W. and Saunders, S.C. (1969) A New Family of Life Distributions. Journal of Applied Probability, 6, 319-327. https://doi.org/10.2307/3212003

[78] Camazine, S., Deneubourg, J.-L., Franks, N.R., Sneyd, J., Theraulaz, G. and Bonabeau, E. (2003) Self-Organization in Biological Systems. Princeton Studies in Complexity, Princeton Univ. Press, Princeton, Oxford.

[79] May, K.A. and Solomon, J.A. (2013) Four Theorems on the Psychometric Function. PLOS ONE, 8, e74815. https://doi.org/10.1371/journal.pone.0074815

[80] Weibull, W. (1939) The Statistical Theory of the Strength of Materials. Ingeniors Vetenskaps Academy Handlingar, 151-153, 45-55.

[81] Weibull, W. (1951) The Statistical Distribution Function of Wide Applicability. Journal of Applied Mechanics, 8, 293-297.

[82] Brown, W.K. and Wohletz, K.H. (1995) Derivation of the Weibull Distribution Based on Physical Principles and Its Connection to the Rosin-Rammler and Lognormal Distributions. Journal of Applied Physics, 78, 2758-2764. https://doi.org/10.1063/1.360073

[83] Batdorf, S.B. (1978) Fundamentals of the Statistical Theory of Fracture. In: Bradt, R.C., Hasselman, D.P.H. and Lange, F.F., Eds., Fracture Mechanics of Ceramics, Vol. 3, Plenum Press, New York, 1.

[84] Gompertz, B. (1825) On the Nature of the Function Expressive of the Law of Hu- 
man Mortality and on a New Mode of Determining the Value of Life Contingencies. Philosophical Transactions of the Royal Society of London, 115, 513-585. https://doi.org/10.1098/rstl.1825.0026

[85] Gavrilov, L.A. and Gavrilova, N.S. (2001) The Reliability Theory of Aging and Longevity. Journal of Theoretical Biology, 213, 527-545. https://doi.org/10.1006/jtbi.2001.2430

[86] Wilson, D.L. (1994) The Analysis of Survival (Mortality) Data: Fitting Gompertz, Weibull, and Logistic Functions. Mechanisms of Ageing and Development, 74, 15-33. https://doi.org/10.1016/0047-6374(94)90095-7

[87] Pham, H. (2008) Mortality Modeling Perspectives. In: Recent Advances in Reliability and Quality in Design, Springer Series in Reliability Engineering, Springer-Verlag, London, 509-516. https://doi.org/10.1007/978-1-84800-113-8_25

[88] Waliszewski, P. and Konarski, J. (2003) The Gompertzian Curve Reveals Fractal Properties of Tumor Growth. Chaos, Solitons and Fractals, 16, 665-674. https://doi.org/10.1016/S0960-0779(02)00469-1

[89] Bru, A., Albertos, S., Subiza, J.L., García-Asenjo, J.L. and Bru, I. (2003) The Universal Dynamics of Tumor Growth. Biophysical Journal, 85, 2948-2961. https://doi.org/10.1016/S0006-3495(03)74715-8

[90] Demicheli, R., Biganzoli, E., Boracchi, P., Greco, M., Hrushesky, W.J.M. and Retsky, M.W. (2006) Allometric Scaling Law Questions the Traditional Mechanical Model for Axillary Lymph Node Involvement in Breast Cancer. Journal of Clinical Oncology, 24, 4391-4396. https://doi.org/10.1200/JCO.2006.05.5988

[91] Oguntunde, P.E., Balogun, O.S., Okagbue, H.I. and Bishop, S.A. (2015) The WeibullExponential Distribution: Its Properties and Applications. Journal of Applied Sciences, 15, 1305-1311. https://doi.org/10.3923/jas.2015.1305.1311

[92] El-Bassiouny, A.H., El-Damcese, M.A., Mustafa, A. and Eliwa, M.S. (2017) Exponentiated Generalized Weibull-Gompertz Distribution with Application in Survival Analysis. Journal of Statistics Applications \& Probability, 6, 7-16. https://doi.org/10.18576/jsap/060102

[93] Ricklefs, R.E. and Scheuerlein, A. (2002) Biological Implications of the Weibull and Gompertz Models of Aging. Journal of Gerontology. Biological Sciences, 57A, B69B76. https://doi.org/10.1093/gerona/57.2.B69

[94] Waliszewski, P. and Konarski, J. (2005) A Mystery of the Gompertz Function. In: Losa, G.A., Merlini, D., Nonnenmacher, T.F. and Weibel, E.R., Eds., Fractals in Biology and Medicine, Birkhäuser, Basel, 277-286.

https://doi.org/10.1007/3-7643-7412-8_27

[95] Watts, D.J. and Strogatz, S.H. (1998) Collective Dynamics of "Small-World" Networks. Nature, 393, 440-442. https://doi.org/10.1038/30918

[96] Nijhout, H.F. (2011) Dependence of Morphometric Allometries on the Growth Kinetics of Body Parts. Journal of Theoretical Biology, 288, 35-43. https://doi.org/10.1016/j.jtbi.2011.08.008

[97] Nijhout, H.F. and German, R.Z. (2012) Developmental Causes of Allometry: New Models and Implications for Phenotypic Plasticity and Evolution. Integrative and Comparative Biology, 52, 43-52. https://doi.org/10.1093/icb/ics068

[98] West, G.B., Brown, J.H. and Enquist, B.J. (2001) A General Model for Ontogenetic Growth. Nature, 413, 628-631. https://doi.org/10.1038/35098076

[99] Hajian-Tilaki, K.O., Hanley, J.A., Joseph, L. and Collet, J.-P. (1977) A Comparison of Parametric and Nonparametric Approaches to ROC Analysis of Quantitative 
Diagnostic Tests. Medical Decision Making, 17, 94-102. https://doi.org/10.1177/0272989X9701700111

[100] Bex, P.J., Metha, A.B. and Makous, W. (1998) Psychophysical Evidence for a Functional Hierarchy of Motion Processing Mechanisms. Journal of the Optical Society of America A, 15, 769-777. https://doi.org/10.1364/JOSAA.15.000769

[101] Pelli, D.G. and Farell, B. (1995) Psychophysical Methods. In: Bass, M., Van Stryland, E.W., Williams, D.R. and Wolfe, W.L., Eds., Handbook of Optics, 2nd Edition, McGraw-Hill, New York, I (29.21-29.13).

[102] Wang, H., Wang, Z., Li, X., et al. (2011) A Robust Approach Based on Weibull Distribution for Clustering Gene Expression Data. Algorithms for Molecular Biology,

6, 14. https://doi.org/10.1186/1748-7188-6-14

[103] Piantanell, L. (1986) A Mathematical Model of Survival Kinetics. I. Theoretical Basis. Archives of Gerontology and Geriatrics, 5, 107-118. https://doi.org/10.1016/0167-4943(86)90014-2

[104] Economos, E.C. (1982) Rate of Aging, Rate of Dying and the Mechanism of Mortality. Archives of Gerontology and Geriatrics, 1, 3-27. https://doi.org/10.1016/0167-4943(82)90003-6

[105] Weon, B.M. and Je, J.H. (2010) Predicting Human Lifespan Limits. Scientific Research, 2, 984-989. https://doi.org/10.4236/ns.2010.29120

[106] Weon, B.M. and Je, J.H. (2011) Plasticity and Rectangularity in Survival Curves. Scientific Reports, 1, Article No. 104. https://doi.org/10.1038/srep00104

[107] Pugno, N.M. (2007) A Statistical Analogy between Collapse of Solids and Death of Living Organisms: Proposal for a "Law of Life". Medical Hypotheses, 69, 441-447. https://doi.org/10.1016/j.mehy.2006.10.067

[108] Pugno, N.M. (2005) On the Statistical Law of Life. Department of Structural Engineering, Politecnico di Torino, Torino.

[109] Stehlik, M., Hermann, P. and Nicolis, O. (2016) Fractal Based Cancer Modelling. REVSTAT-Statistical Journal, 14, 139-155.

[110] Liu, S., Wang, Y., Xu, K., Wang, Z., Fan, X., Zhang, C., Li, S., Qiu, X. and Jiang, T. (2017) Relationship between Necrotic Patterns in Glioblastoma and Patient Survival: Fractal Dimension and Lacunarity Analyses Using Magnetic Resonance Imaging. Scientific Reports, 7, Article No. 8302. https://doi.org/10.1038/s41598-017-08862-6

[111] Stehlik, M., Wartner, F. and Minarova, M. (2013) Fractal Analysis for Cancer Research: Case Study and Simulation of Fractals. Pliska Studia Mathematica Bulgarica, 22, 195-206.

[112] Weston, C.L., Douglas, C., Craft, A.W., Lewis, I.J. and Machin, D. (2004) Establishing Long-Term Survival and Cure in Young Patients with Ewing's Sarcoma. British Journal of Cancer, 91, 225-232. https://doi.org/10.1038/sj.bjc.6601955

[113] Zhang, Z.-H. (2016) Parametric Regression Model for Survival Data: Weibull Regression Model as an Example. Annals of Translational Medicine, 4, 484. https://doi.org/10.21037/atm.2016.08.45

[114] Jones, G. and Rocke, D.M. (2002) Multivariate Survival Analysis with DoublyCensored Data: Application to the Assessment of Accutane Treatment for Fibrodysplasia Ossificans Progressive. Statistics in Medicine, 21, 2547-2562. https://doi.org/10.1002/sim.1123

[115] Pourhoseingholi, M.A., Pourhoseingholi, A., Vahedi, M., Moghimi Dehkordi, B., Safaee, A., Ashtari, S. and Zali, M.R. (2011) Alternative for the Cox Regression 
Model: Using Parametric Models to Analyze the Survival of Cancer Patients. Iranian Journal of Cancer Prevention, 4, 1-9.

[116] Pourhoseingholi, M., Hajizadeh, E., Moghimi Dehkordi, B., Safaee, A., Abadi, A. and Zali, M.R. (2007) Comparing Cox Regression and Parametric Models for Survival of Patients with Gastric Carcinoma. Asian Pacific Journal of Cancer Prevention: $A P J C P, 8,412-416$.

[117] Hoyle, M.W. and Henley, W. (2011) Improved Curve Fits to Summary Survival Data: Application to Economic Evaluation of Health Technologies. BMC Medical Research Methodology, 11, 139. https://doi.org/10.1186/1471-2288-11-139

[118] Barriga, G.D.C., Louzada-Neto, F., Ortega, E.M.M. and Cancho, V. (2010) A Bivariate Regression Model for Matched Paired Survival Data: Local Influence and Residual Analysis. Statistical Methods and Applications, 19, 477-495.

https://doi.org/10.1007/s10260-010-0140-1

[119] Andersson, T. (2007) Analyzing Time Trends in Cancer Patient Survival Using Cure Fraction Models. U.U.D.M. Project Report 4, Department of Mathematics, Uppsala University, Uppsala.

[120] Fuller, A.F. and Griffiths, C.M. (1983) Gynecologic Oncology. M. Nijhoff, The Hague. https://doi.org/10.1007/978-1-4613-3852-9

[121] Lambert, P.C., Thompson, J.R., Weston, C.L. and Dickman, P.W. (2007) Estimating and Modeling the Cure Fraction in Population-Based Cancer Survival Analysis. Biostatistics, 8, 576-594. https://doi.org/10.1093/biostatistics/kxl030

[122] Cover, T.M. and Thomas, J.A. (2005) Elements of Information Theory. Wiley, Hoboken. https://doi.org/10.1002/047174882X

[123] Szasz, A., Dani, A., Varkonyi, A. and Magyar, T. (2005) Retrospective Analysis of 1180 Oncological Patients Treated by Electro-Hyperthermia in Hungary. Strahlentherapy Onkologie (Radiation Oncology), 181, 121-122.

[124] Szasz, A., Szasz, N. and Szasz, O. (2010) Oncothermia: Principles and Practices. Springer Science, Heidelberg, Ch. 4, 295-302. https://doi.org/10.1007/978-90-481-9498-8 